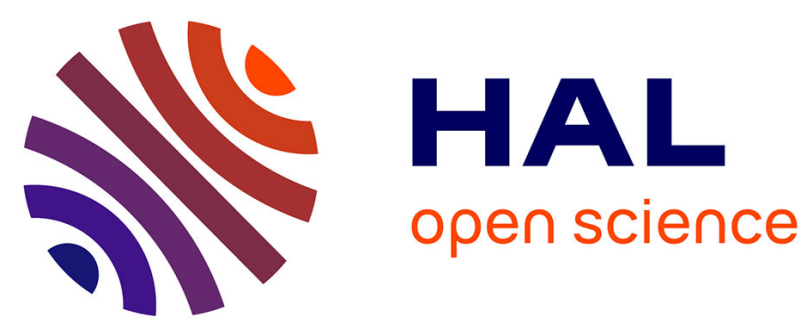

\title{
Estimation of time delay and interface roughness by GPR using modified MUSIC
}

Meng Sun, Cédric Le Bastard, Nicolas Pinel, Yide Wang, Jianzhong Li, Jingjing Pan, Zhiwen Yu

\section{- To cite this version:}

Meng Sun, Cédric Le Bastard, Nicolas Pinel, Yide Wang, Jianzhong Li, et al.. Estimation of time delay and interface roughness by GPR using modified MUSIC. Signal Processing, 2017, 132, pp.272 283. 10.1016/j.sigpro.2016.05.029 . hal-01400510

HAL Id: hal-01400510 https://hal-univ-rennes1.archives-ouvertes.fr/hal-01400510

Submitted on 22 Nov 2016

HAL is a multi-disciplinary open access archive for the deposit and dissemination of scientific research documents, whether they are published or not. The documents may come from teaching and research institutions in France or abroad, or from public or private research centers.
L'archive ouverte pluridisciplinaire HAL, est destinée au dépôt et à la diffusion de documents scientifiques de niveau recherche, publiés ou non, émanant des établissements d'enseignement et de recherche français ou étrangers, des laboratoires publics ou privés. 


\title{
Estimation of time delay and interface roughness by GPR using modified MUSIC
}

\author{
Meng Sun ${ }^{\mathrm{a}}$, Cédric Le Bastard ${ }^{\mathrm{b}, \mathrm{a}}$, Nicolas Pinel ${ }^{\mathrm{c}}$, Yide Wang ${ }^{\mathrm{a}}$, Jianzhong Li ${ }^{\mathrm{a}, \mathrm{d}}$, \\ Jingjing $\mathrm{Pan}^{\mathrm{a}}$, Zhiwen $\mathrm{Yu}^{\mathrm{d}}$ \\ anstitut d'Electronique et Télécommunications de Rennes (IETR), LUNAM Université \\ Université de Nantes, UMR CNRS 6164, Rue Christian Pauc BP 50609, Nantes 44306, France \\ Email:(jianzhong.li@etu.univ-nantes.fr) \\ ${ }^{b}$ Cerema (Centre for expertise and engineering on risks, environment, mobility, urban and country \\ planning), 23 avenue de l'Amiral Chauvin, BP 69, 49136 Les Ponts de Cé, France. \\ ${ }^{c}$ Alyotech, 2 rue Antoine Becquerel, 35700 Rennes, France. \\ ${ }^{d}$ South China University of Technology, Guangzhou 510641, Peoples Republic of China
}

\begin{abstract}
In civil engineering, roadway structure evaluation is an important application which can be carried out by ground penetrating radar. In this paper, firstly a signal model taking into account the influence of interfaces roughness (surface and interlayer) is proposed. In order to estimate the time delay and interface roughness, we propose a method composed of 2 steps: 1) a modified MUSIC algorithm is proposed for time delay estimation; 2) the interface roughness is estimated by using Maximum Likelihood method (MLE) with the estimated time delays. The proposed algorithms are tested on data obtained by a method of moments (MoM). Numerical examples are provided to demonstrate the performance of the proposed algorithm. Keywords: Ground Penetrating Radar (GPR), Time-Delay Estimation (TDE), Roughness, modified MUSIC, Method of Moments (MoM), Maximum Likelihood method (MLE).
\end{abstract}




\section{Introduction}

Ground penetrating radar (GPR) is widely used as a non destructive testing technique for road pavement survey $[1,2,3,4,5,6]$, particularly for the measurement of different layer thicknesses. In road pavement survey, the road layers are assumed 5 to be horizontally stratified [7]. Useful information about the vertical structure of the roadway can then be extracted from radar profiles by means of echo detection and amplitude estimation $[8,9,10,11]$. Echo detection provides the time-delay estimation (TDE) associated with each interface, while amplitude estimation allows retrieving the wave speed within each layer. In this paper, we focus on the practical case when the backscattered echoes are overlapped $[12,13]$, which means that the thickness is smaller than the wavelength in the medium. In this case, high resolution and superresolution methods $[14,15,16,17]$ (or subspace methods) can be used to estimate the time delays of echoes and then to measure the small pavement thicknesses (with estimated permittivity). However, these methods assume that the interfaces of the layers are flat. For decimetre-scale GPR wavelengths (in the air), this assumption can be held, but for an ultra-wide band radar, this is no longer suitable. The influence of interface roughness and heterogeneous of medium must then be analysed $[13,18,19]$. In this paper, only the interface roughness is discussed. For large frequency bands, the case of a heterogeneous medium case can be considered as a homogeneous medium with an equivalent permittivity. The heterogeneity medium will be studied in future work. The interface roughness is characterized by a particular frequency signature of echoes amplitudes, which is decreasing with frequency. In this paper, we propose to firstly estimate the time delays and then the interface roughness with an ultra-wideband GPR. In the following, the media are 
assumed to be lossless [20, 21]. Roughness parameter is important for road safety, like pavement skid resistance analysis, and for analysing the inside of the pavement, especially to detect the cracks or debondings by highlighting the disaggregation of interface materials.

In $[19,20]$, this kind of work has already been carried out, but the frequency behaviour coming from the roughness has been simply approximated by an exponential function (using a curve fitting method). In this situation, the high resolution methods can easily be applied for parameters estimation (time delays and roughness). Nevertheless, it is suitable only for narrow band (less than $2 \mathrm{GHz}$ ). With the widening of the frequency band, the curve fitting error will increase rapidly, which may bring errors to the interface roughness estimation. In order to reduce the errors coming from the curve fitting, we propose a modified MUSIC algorithm which can take into account several possible frequency behaviours, more adaptable for ultrawideband GPR. Like in [19, 20], we also focus on the estimation of time delays and interface roughness. Unlike the methods in $[19,20]$, the proposed method allows estimating the time delays without knowing the frequency behaviour from roughness. Then, the roughness parameter is estimated by MLE [22] with the estimated time delays from the modified MUSIC algorithm. This step uses a model of which the roughness frequency behaviour is approximated as a Gaussian function. The performance of the proposed algorithm is tested on data simulated from PILE method $[13,23,24,25]$, which is based on the method of moments.

This paper is organised as follows. Section 2 gives the simulation results of the scattering of EM waves from random rough interfaces, and studies the frequency behaviour of the backscattered echoes. In section 3, the radar data model and 
preprocessing methods are presented. In section 4, a modified MUSIC algorithm is proposed to estimate the time delays without knowing the frequency behaviour. Moreover, the roughness parameters are estimated by MLE [22]. Simulation results and a discussion on the performance of the proposed algorithm are given in section 5. Finally, conclusions and perspectives are drawn.

\section{Rough pavement scattering model}

In order to give some ideas about the scattering from a rough pavement, a realistic simulation of a typical thin asphalt road structure (pavement layer) is considered. The rigorous electromagnetic method PILE (propagation inside layer expansion) $[23,25]$ based on the MoM, provides simulation data that allows showing the influence of the interface roughness on the backscattered echoes of stratified media. The simulation parameters are chosen to match the air-coupled radar configuration at vertical incidence that is used for pavement survey at traffic speed; the probing scope is assumed to be limited to the first two layers of the pavement structure. The considered pavement structure is an Ultra-Thin Asphalt Surfacing (UTAS), which is made of a layer medium $\Omega_{2}$ with mean thickness $\bar{H}=20 \mathrm{~mm}$. For the rolling band (or base band) corresponding to the medium $\Omega_{3}$, it has the same composition as the medium $\Omega_{2}$, see Fig. 1. We assume that $\Omega_{2}$ and $\Omega_{3}$ are homogeneous media for a normal incidence angle $\left(\theta_{i}=0\right.$ in Fig. 1$)$ and the frequency band under study is $[0.5,10.5] \mathrm{GHz}$. For the considered media, their relative permittivity $\varepsilon_{r}$ typically ranges between 4 and 8 . Moreover, in this paper, the media are assumed to be lossless. For the simulations, we take $\varepsilon_{r 2}=4.5$ and $\varepsilon_{r 3}=7$. The two rough interfaces $\Sigma_{A}$ and $\Sigma_{B}$ are assumed to have a Gaussian height probability density function and an 
exponential height autocorrelation function $[26,27]$. For $\Sigma_{A}$, the RMS height $\sigma_{h A}$ is about $0.6-1 \mathrm{~mm}$, and the correlation length $L_{c A}$ is about $5-10 \mathrm{~mm}[26,27]$. For $\Sigma_{B}$, the RMS height $\sigma_{h B}$ and the correlation length $L_{c B}$ are greater than those of $\Sigma_{A}$. It is assumed that the antenna radiates a vertically polarized plane wave in far field of probed pavement (in practice, the antenna is about $400 \mathrm{~mm}$ above the rough surface). The typical width of a probed surface antenna footprint is about $300-500 \mathrm{~mm}$. In the simulations, we consider surfaces of length $L=2400 \mathrm{~mm}$, illuminated by a Thorsos beam (the Thorsos beam is a tapered plane wave, whose tapering has a Gaussian shape; the tapering is used to reduce the incident field to near zero at the edges of the surface realisations and thereby to reduce edge effects to negligible levels) of attenuation parameter $g=L / 8$. The two rough interfaces are sampled with a sampling step $\Delta x=\operatorname{Re}\left(\lambda_{2}\right) / 8$, where $\lambda_{2}$ is the wavelength inside $\Omega_{2}$ with $\lambda_{2}=\lambda_{0} / \sqrt{\varepsilon_{r 2}}\left(\lambda_{0}\right.$ is the wavelength in vacuum) and $\operatorname{Re}(\ldots)$ is the real part. We take an incident wave with normal incidence $\left(\theta_{i}=0\right)$, and then calculate the first two scattered echoes $s_{1}$ and $s_{2}$ from the scattered field.

In order to investigate the influence of interface roughness on the backscattered echoes, a rough pavement is tested, with roughness parameters $\sigma_{h A}=1.0 \mathrm{~mm}$, $L_{c A}=6.4 \mathrm{~mm}, \sigma_{h B}=2.0 \mathrm{~mm}, L_{c B}=15 \mathrm{~mm}$. According to the selected parameters, PILE method provides the first two backscattered echoes $s_{1}$ and $s_{2}$ at each frequency over the frequency band $f \in[0.5,10.5] \mathrm{GHz}$, with sampling step $\Delta f=0.1$ GHz. Simulated data were obtained by a Monte Carlo process with 100 independent realizations. Two models are presented: an exponential shape and a Gaussian shape. For doing so, a curve fitting is made by using least squares method to estimate the parameters of the model. In $[19,20]$, the exponential shape $|s(f)|=s_{k} \times \exp (-\bar{b} f)$ 
with roughness parameter $\bar{b}$ of the scattered echo amplitude is studied. In this paper, we introduce a Gaussian shape $|s(f)|=s_{k} \times \exp \left(-b f^{2}\right)$ with a different roughness parameter $b$; where $s_{k}$ is the amplitude of the considered backscattered echo in the flat pavement.

Curve fittings are made with radar data from PILE in 3 narrow bands $(f \in$ $[0.5,1.5] \mathrm{GHz}, f \in[0.5,2.5] \mathrm{GHz}, f \in[0.5,3.5] \mathrm{GHz})$ and 3 large frequency bands $(f \in[0.5,6.5] \mathrm{GHz}, f \in[0.5,8.5] \mathrm{GHz}, f \in[0.5,10.5] \mathrm{GHz})$. Figs. 2 and 3 give the frequency behaviour of the backscatter echoes. We can notice that the amplitudes of the backscattered echoes are decreasing when the frequency increases, especially for the second echo. Thus, the influence of the interface roughness cannot be neglected any more, the frequency behaviour of the echoes should be considered in radar data processing. In addition, Figs. 2 and 3 also show the curve fitting results of the Gaussian and exponential functions. It can be noticed that, the Gaussian fitting is generally in good agreement with radar data in the whole frequency band, but for the exponential fitting, significant deviations can be found, particularly for large frequency bands. We assess the performance of the curve fitting by Root Means Square Errors (RMSE), as shown in Table. 1. The results are in agreement with the above figures, the Gaussian fitting is more precise than the exponential fitting.

\section{Signal model}

In the previous section, the frequency behaviour of interface roughness has been studied by using PILE method. In this paper, we focus on the first two or three top layers of roadway, the whole thickness (of the roadway) is about 6 to $13 \mathrm{~cm}$. Indeed, in our study, we focus on pavements which are composed of an ultra-thin 
asphalt surface (1 to $3 \mathrm{~cm}$ thickness) and a base course (5 to $10 \mathrm{~cm}$ thickness). For pavement materials, according to the data provided in [28], the conductivity usually ranges within the interval $\left[10^{-3} ; 10^{-2}\right] \mathrm{S} / \mathrm{m}$. Thus, the media considered in this paper are assumed to be low-loss media. Moreover, the pavement permittivity remains constant within the GPR bandwidth and generally ranges between 4 and 8 . Thus, the considered media are low-loss and non-dispersive media. In addition, the dispersivity of the medium can be neglected [29], if the surface medium is slightly lossy. For flat pavements, the backscattered echoes can be simply considered as timeshifted and attenuated copies of the transmitted signal $[9,12,21,30]$. For a rough pavement, a new signal model is presented with roughness for non-dispersive media and without considering the conductivity as follows:

$$
r\left(f_{i}\right)=\sum_{k=1}^{K} e\left(f_{i}\right) s_{k} w_{k}\left(f_{i}\right) \exp \left(-j 2 \pi f_{i} t_{k}\right)+n\left(f_{i}\right)
$$

115

where

- $K$ is the number of interfaces;

- $e\left(f_{i}\right)$ is the radar pulse at frequency $f_{i}$;

- $s_{k}$ represents the reflection coefficient of the $k$ th scattered echo with flat interfaces, which is independent of $f_{i}$;

- $n\left(f_{i}\right)$ is an additive white Gaussian noise, with zero mean and variance $\sigma^{2}$;

- $w_{k}\left(f_{i}\right)$ represents the frequency behaviour of the $k$ th scattered echo at frequency $f_{i}=f_{1}+(i-1) \Delta f$ and $i=1,2 \ldots N, N$ being the number of used frequencies; 
$f_{1}$ is the lowest frequency of the studied frequency band and $\Delta f$ is the frequency step.

Eq. (1) can be written in the following vector form:

$$
\mathbf{r}=\Lambda \mathrm{As}+\mathbf{n}
$$

with the following notation definitions:

- $\mathbf{r}=\left[r\left(f_{1}\right) r\left(f_{2}\right) \cdots r\left(f_{N}\right)\right]^{T}$ is the $(N \times 1)$ received signal vector, called observation vector, which may represent either the Fourier transform of the GPR signal or the measurements from a step frequency radar; the superscript $T$ denotes the transpose operator;

- $\boldsymbol{\Lambda}=\operatorname{diag}\left(e\left(f_{1}\right), e\left(f_{2}\right), \ldots, e\left(f_{N}\right)\right)$ is a $(N \times N)$ diagonal matrix, whose diagonal elements are the Fourier transform $e(f)$ of the radar pulse $e(t)$;

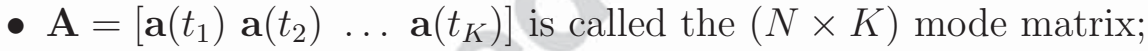

- $\mathbf{a}\left(t_{k}\right)=\left[\exp \left(-2 j \pi f_{1} t_{k}\right) w_{k}\left(f_{1}\right) \exp \left(-2 j \pi f_{2} t_{k}\right) w_{k}\left(f_{2}\right)\right.$

$\left.\ldots \exp \left(-2 j \pi f_{N} t_{k}\right) w_{k}\left(f_{N}\right)\right]^{T}$ is the mode vector;

- $\mathbf{s}=\left[\begin{array}{llll}s_{1} & s_{2} & \cdots & s_{K}\end{array}\right]^{T}$ is the $(K \times 1)$ vector of echoes amplitudes in the case of flat interfaces;

- $\mathbf{n}=\left[n\left(f_{1}\right) n\left(f_{2}\right) \cdots n\left(f_{N}\right)\right]^{T}$ is the $(N \times 1)$ noise vector, with variance matrix $\sigma^{2} \mathbf{I}$ 
According to signal model (2) and assuming that the noise is independent of the echoes, the covariance matrix $\mathbf{Y}$ can be written as:

$$
\begin{aligned}
\mathbf{Y} & =E\left(\mathbf{r r}^{H}\right)=\mathbf{\Lambda} \mathbf{A} E\left(\mathbf{s s}^{H}\right) \mathbf{A}^{H} \boldsymbol{\Lambda}^{H}+E\left(\mathbf{n n}^{H}\right) \\
& =\mathbf{\Lambda} \mathbf{A} \mathbf{S} \mathbf{A}^{H} \boldsymbol{\Lambda}^{H}+\sigma^{2} \mathbf{I}
\end{aligned}
$$

where $E(:)$ denotes the ensemble average, $\mathbf{S}$ is the $K \times K$ dimensional covariance matrix of the source vector $\mathbf{s}$ and $\mathbf{I}$ is an identity matrix. In the following, the data are divided by the pulse, thus the new observation vector $\mathbf{r}^{\prime}$ can be written as $\mathbf{r}^{\prime}=\mathbf{\Lambda}^{-1} \mathbf{r}=\mathbf{A} \mathbf{s}+\mathbf{\Lambda}^{-1} \mathbf{n}=\mathbf{A} \mathbf{s}+\mathbf{b}$, where $\mathbf{b}$ is the new noise vector after division. Thus, the new covariance matrix $\mathbf{R}_{0}$ can be written as:

$$
\mathbf{R}_{0}=E\left(\mathbf{r}^{\prime} \mathbf{r}^{\prime H}\right)=\boldsymbol{\Lambda}^{-1} \mathbf{Y} \boldsymbol{\Lambda}^{-H}=\mathbf{A} \mathbf{S} \mathbf{A}^{H}+\sigma^{2} \mathbf{\Sigma}
$$

with

$$
\boldsymbol{\Sigma}=\boldsymbol{\Lambda}^{-1} \boldsymbol{\Lambda}^{-H}=\operatorname{diag}\left(\frac{1}{\left|e\left(f_{1}\right)\right|^{2}}, \frac{1}{\left|e\left(f_{2}\right)\right|^{2}}, \cdots, \frac{1}{\left|e\left(f_{N}\right)\right|^{2}}\right)
$$

In practice, the correlation between echoes degrades the subspace algorithm's performance. In this situation, preprocessing methods like spatial smoothing technique are used to obtain a new covariance matrix of restored rank. This kind of techniques only works on uniform linear frequency behaviours [31]. As the frequency behaviour of backscattered echoes $w(f)$ can have an arbitrary frequency behaviour, methods like spatial smoothing technique cannot be used directly. In order to solve this problem, we propose to interpolate the frequency behaviour of backscattered 
echoes into a uniform linear. Then, the spatial smoothing technique can be applied. This kind of algorithms is called interpolated spatial smoothing technique [32, 33]. By using interpolation, a new covariance matrix can be written as follows:

$$
\overline{\mathbf{R}}=\mathbf{B} \mathbf{A S A} \mathbf{A}^{H} \mathbf{B}^{H}+\sigma^{2} \mathbf{B} \boldsymbol{\Sigma} \mathbf{B}^{H}
$$

where $\mathbf{B}$ is a transformation matrix of interpolation (the details of the interpolation are provided in appendix A). In the following section, a modified MUSIC algorithm is proposed and applied for time delay estimation. This method assumes that the noise is a Gaussian white noise. To ensure this condition, like in [20], the noise covariance matrix should be removed. As the radar pulse (measured by the echo backscattered from a metallic plane) and the transformation matrix $\mathbf{B}$ are known, and the noise variance $\sigma^{2}$ is estimated by the propagator method [34], the new noise free covariance matrix $\mathbf{R}$ can be written as follows:

$$
\mathbf{R}=\mathbf{B} \mathbf{A} \mathbf{S A}^{H} \mathbf{B}^{H}+0 \times \mathbf{I} \approx \overline{\mathbf{R}}-\hat{\sigma}^{2} \mathbf{B} \boldsymbol{\Sigma} \mathbf{B}^{H}
$$

where $\hat{\sigma}^{2}$ is the estimated noise variance. Then, the spatial smoothing preprocessing technique can be applied [35]. The SSP technique estimates the modified covariance matrix $\mathbf{R}_{S S P}$ as follows [35]:

$$
\mathbf{R}_{S S P}=\frac{1}{M} \sum_{k=1}^{M} \mathbf{R}_{k}
$$

where $\mathbf{R}_{k}$ is the $k^{t h}$ sub-band of the covariance matrix $\mathbf{R}, N$ frequencies, $M$ overlapping sub-bands of length $L$ are considered. $N, M$ and $L$ are related to one another 
by:

$$
N=L+M-1
$$

\section{Time delay and interface roughness estimation}

When the interface roughness is taken into account, high resolution algorithms like MUSIC or ESPRIT cannot be used directly in theory, due to the unknown frequency behaviour $w_{k}\left(f_{i}\right)$ of the echoes. Therefore, we propose a modified MUSIC algorithm to estimate the time delays, and then MLE is used to estimate the interface roughness.

\subsection{Modified MUSIC algorithm}

In this section, a modified MUSIC algorithm is proposed, which allows estimating only the time delays. The mode vector a can be written as follows:

$$
\begin{aligned}
\mathbf{a}(t) & =\left[\exp \left(-2 j \pi f_{1} t\right) \bar{w}\left(f_{1}\right) \exp \left(-2 j \pi f_{2} t\right) \bar{w}\left(f_{2}\right)\right. \\
& \left.\ldots \exp \left(-2 j \pi f_{L} t\right) \bar{w}\left(f_{L}\right)\right]^{T} \\
& =\operatorname{diag}\left\{\exp \left(-2 j \pi f_{1} t\right), \exp \left(-2 j \pi f_{2} t\right) \ldots, \exp \left(-2 j \pi f_{L} t\right)\right\} \\
& {\left[\bar{w}\left(f_{1}\right) \bar{w}\left(f_{2}\right) \ldots \bar{w}\left(f_{L}\right)\right]^{T} } \\
& =\widehat{\mathbf{A}} \mathbf{k}
\end{aligned}
$$

where $\widehat{\mathbf{A}}=\operatorname{diag}\left\{\exp \left(-2 j \pi f_{1} t\right), \exp \left(-2 j \pi f_{2} t\right), \ldots, \exp \left(-2 j \pi f_{L} t\right)\right\}$ and $\mathbf{k}=\left[\bar{w}\left(f_{1}\right) \bar{w}\left(f_{2}\right) \ldots \bar{w}\left(f_{L}\right)\right]^{T}$ with $\bar{w}(f)$ the frequency behaviour of the backscattered echoes after interpolation. $\mathbf{k}$ is a real vector. 
The pseudo-spectrum of MUSIC can be written as:

$$
P(t)=\left[\min _{k}\left\{\frac{\mathbf{k}^{H} \widehat{\mathbf{A}}^{H} \mathbf{U}_{N} \mathbf{U}_{N}^{H} \widehat{\mathbf{A}} \mathbf{k}}{\mathbf{k}^{H} \widehat{\mathbf{A}}^{H} \widehat{\mathbf{A}} \mathbf{k}}\right\}\right]^{-1}
$$

150 eigenvectors. Referring to [36], $P(t)$ is equal to the minimum generalized eigenvalue $\lambda_{\text {min }}$ of $\widehat{\mathbf{A}}^{H} \mathbf{U}_{\mathbf{N}} \mathbf{U}_{N}^{H} \widehat{\mathbf{A}}$ and $\widehat{\mathbf{A}}^{H} \widehat{\mathbf{A}}$, satisfying (with $\mathbf{k}_{\text {min }}$ the corresponding generalized eigenvector):

$$
\widehat{\mathbf{A}}^{H} \mathbf{U}_{N} \mathbf{U}_{N}^{H} \widehat{\mathbf{A}} \mathbf{k}_{\min }=\lambda_{m i n} \widehat{\mathbf{A}}^{H} \widehat{\mathbf{A}} \mathbf{k}_{\min }=\lambda_{\min } \mathbf{k}_{\text {min }}
$$

The pseudo-spectrum of MUSIC can also be written as the reciprocal of the minimum eigenvalue of real $\left\{\widehat{\mathbf{A}}^{H} \mathbf{U}_{N} \mathbf{U}_{N}^{H} \widehat{\mathbf{A}}\right\}[36,37]$ :

$$
P(t)=\frac{1}{\lambda_{\min }(t)}
$$

By using (11), we need only to search the spectrum in the time domain without knowing the influence of the frequency behaviour. Nevertheless, it has a false peak in the middle of two true values. For example, when two echoes are considered, we assume that $t_{1}$ and $t_{2}\left(t_{2}>t_{1}\right)$ are the time delays of the echoes, then we can prove that $t_{3}=\frac{t_{2}-t_{1}}{2}$ is also a solution of $\lambda_{\min }(t)=0$ (the proof is given in appendix $\mathrm{B}$ ). In [37], based on the characteristics of $\lambda_{\min }(t)$ corresponding to the false time delay and the true time delays, they propose a new pseudo-spectrum of MUSIC to cancel 
the false time delay, which can be expressed as follows:

$$
P(t)=10 \log _{10}\left\{\frac{\lambda_{1}(t)}{\lambda_{2}(t)}\right\}
$$

where $\lambda_{k}(t)$ is the $k$ th eigenvalue of real $\left\{\widehat{\mathbf{A}}^{H} \mathbf{U}_{N} \mathbf{U}_{N}^{H} \widehat{\mathbf{A}}\right\}$, and $\lambda_{L}(t) \geq \lambda_{L-1}(t) \geq$ $\ldots \geq \lambda_{1}(t)$. Still, the above method only works for the case of two echoes. Indeed, for the case where the number of echoes is superior to 2, eq. (12) does not work. For example, when a true time delay has the same value as a false one, this true time delay will also be cancelled. From appendix B, we show that the number of zero eigenvalues of real $\left\{\widehat{\mathbf{A}}^{H} \mathbf{U}_{N} \mathbf{U}_{N}^{H} \widehat{\mathbf{A}}\right\}$ corresponding to the true time delay is odd, and to the false time delay, this number is even. Based on the above characteristics, we propose a generalized pseudo-spectrum for modified MUSIC as follows:

$$
P(t)=\left\{\begin{array}{l}
\frac{\lambda_{2}(t)}{\lambda_{1}(t)} \frac{\lambda_{4}(t)}{\lambda_{3}(t)} \cdots \frac{\lambda_{L-1}(t)}{\lambda_{L-2}(t)} \quad L=2 n+1 \\
\frac{\lambda_{2}(t)}{\lambda_{1}(t)} \frac{\lambda_{4}(t)}{\lambda_{3}(t)} \cdots \frac{\lambda_{L}(t)}{\lambda_{L-1}(t)} \quad L=2 n
\end{array}\right.
$$

where $n=0,1,2 \ldots$ and $L$ can be an odd or even number. The pseudo-spectrums of MUSIC in (11), (12) and (13) are shown in Fig. 4. In the simulation, 3 time delays (1 ns, $1.3 \mathrm{~ns}$ and $1.6 \mathrm{~ns}$ ) are considered, the second time delay is in the middle of the other two time delays. In order to make a better comparison between the three pseudo-spectrums, an amplitude normalization is made in Fig. 4. Modified MUSIC in (11) obtains two false peaks at $1.15 \mathrm{~ns}$ and $1.45 \mathrm{~ns}$. By using (12), the two false peaks are removed, but also the second time delay. Only the proposed method (13) can successfully remove the false time delays and keep the true time delays. Thus, 
Eq. (13) is used in the following of the paper.

\subsection{MLE for roughness parameter estimation}

For the frequency behaviour of backscattered echoes, it has been found in the preof ultra thin asphalt surfacing with a relative permittivity equal to 4.5 overlying a base band with a relative permittivity equal to 7 . We consider two scattered echoes corresponding to the time delays $1 \mathrm{~ns}$ and $1.3 \mathrm{~ns}$, which corresponds to thickness 
of the first layer of approximately $20 \mathrm{~mm}$ and the second layer is infinite. In the simulations, four pavements are studied (the rough interfaces are assumed to have a Gaussian height probability density function and an exponential height autocorrelation function) $[26,27]$ with different root mean square heights $\sigma_{h}$, correlation lengths $L_{h}$ and conductivities of the layers $\delta$ :

- Case 1. $\sigma_{h A}=1.0 \mathrm{~mm}, L_{c A}=6.4 \mathrm{~mm}, \sigma_{h B}=2.0 \mathrm{~mm}, L_{c B}=15 \mathrm{~mm}$, lossless media.

- Case 2. $\sigma_{h A}=1.0 \mathrm{~mm}, L_{c A}=6.4 \mathrm{~mm}, \sigma_{h B}=2.5 \mathrm{~mm}, L_{c B}=15 \mathrm{~mm}$, lossless media.

- Case 3. $\sigma_{h A}=1.5 \mathrm{~mm}, L_{c A}=6.4 \mathrm{~mm}, \sigma_{h B}=3.0 \mathrm{~mm}, L_{c B}=15 \mathrm{~mm}$, lossless media.

- Case 4. $\sigma_{h A}=1.0 \mathrm{~mm}, L_{c A}=6.4 \mathrm{~mm}, \sigma_{h B}=2.0 \mathrm{~mm}, L_{c B}=15 \mathrm{~mm}$, low-loss media $\left(\delta_{A}=5 \times 10^{-3} \mathrm{~S} / \mathrm{m}, \delta_{B}=10^{-2} \mathrm{~S} / \mathrm{m}\right)$.

When the frequency band is $0.5-3.5 \mathrm{GHz}$, with $0.05 \mathrm{GHz}$ frequency step (61 frequency samples), the echoes are slightly overlapped. When the frequency band is $0.5-6.5 \mathrm{GHz}$, with $0.1 \mathrm{GHz}$ frequency step (61 frequency samples), the echoes are non-overlapped. The covariance matrix is estimated from 1000 independent snapshots. The interpolated SSP technique is used to reduce the cross-correlation between the echoes and the number of sub-bands $(M)$ is equal to 20 . The signal-to-noise ratio (SNR) is defined as the ratio between the powers of the second echo and noise variance. In the first simulation, a fixed $\mathrm{SNR}=20 \mathrm{~dB}$ is used for three different rough pavements. 
Figs. 6-10 show the pseudo-spectrums of modified MUSIC. Two peaks corresponding to the time delays of the first two scattered echoes are well estimated. Simulation results demonstrate that the proposed algorithm can handle cases where both echoes are either overlapped or non-overlapped and for either lossless or lowloss media. The roughness parameters could also be estimated by using the MLE with the estimated time delays (see Figs. 11-15). Table. 2 gives the results of estimated time delays $\left(\hat{t}_{k}\right)$ and estimated roughness parameters $\left(\hat{b}_{k}\right)$. We compare the estimated frequency behaviours with the data from PILE in Figs. 11-15. From the frequency behaviour of the four different cases, it is shown that the expressions of the echoes are in agreement with the data from PILE for various roughness parameters with either lossless media or low-loss media.

Then, in the second simulation, we evaluate the performance of modified MUSIC, which is assessed with a Monte-Carlo process of 500 independent runs of the algorithm with independent noise snapshots and from the RRMSE of the evaluated parameter as follows:

$$
\operatorname{RRMSE}(z)=\frac{\sqrt{\frac{1}{U} \sum_{j=1}^{U}\left(\hat{z}_{j}-z\right)^{2}}}{z},
$$

where $\hat{z}_{j}$ denotes the estimated parameter for the $j$ th run of the algorithm, and $z$ the true value. In the simulation, the parameter $z$ can represent either the first $\left(t_{1}\right)$ or the second $\left(t_{2}\right)$ time delay. Only case 1 is considered. In the time delay estimation, as expected, it can be seen that the RRMSE is continuously decreasing when the SNR increases. Fig. 16 shows that the proposed method gives relatively good performances in time delay estimation. 
In the third simulation, the performance of the proposed method is tested on a pavement which is composed of 3 rough interfaces (four layers). The simulation parameters of the pavement are chosen as follows: the permittivities of first three with coherent backscattered echoes. After applying the interpolated spatial smoothing technique to decorrelate the received echoes, we propose a modified MUSIC algorithm, which is able to estimate the time delays without knowing the frequency behaviour from roughness. Then, the influence of the interface roughness is estimated 
by MLE. These algorithms are applied to evaluate the pavement. The performance of the proposed algorithms is tested on data from MoM. The proposed algorithms show good performance for time delays and interface roughness estimation. In perspective, the proposed method will be extended to dispersive media (soils or hydraulic concretes).

\section{Appendix A}

The mode vector can be written as:

$$
\begin{aligned}
& \mathbf{a}\left(t_{k}\right)=\left[\exp \left(-2 j \pi f_{1} t_{k}\right) w_{k}\left(f_{1}\right) \exp \left(-2 j \pi f_{2} t_{k}\right) w_{k}\left(f_{2}\right) \ldots \exp \left(-2 j \pi f_{N} t_{k}\right) w_{k}\left(f_{N}\right)\right]^{T} \\
& =\operatorname{diag}\left\{w_{k}\left(f_{1}\right), w_{k}\left(f_{2}\right) \ldots w_{k}\left(f_{N}\right)\right\}\left[\exp \left(-2 j \pi f_{1} t_{k}\right), \exp \left(-2 j \pi f_{2} t_{k}\right) \ldots \exp \left(-2 j \pi f_{N} t_{k}\right)\right]^{T} \\
& =\mathbf{C} \overline{\mathbf{a}}
\end{aligned}
$$

The frequency behaviour $w(f)$ depends on the RMS height $\sigma_{h}$ and the correlation length $L_{h}$, thus matrix $\mathbf{C}$ also changes with $\sigma_{h}$ and $L_{h}$, it can be expressed as $\mathbf{C}\left(\sigma_{h}, L_{h}\right)$. We propose to interpolate $w(f)$ into a uniform linear frequency behaviour. The procedure is as follows:

- Define a set of $\sigma_{h}=\left\{\sigma_{h 1}, \sigma_{h 2} \cdots \sigma_{h G}\right\}$ and a set of $L_{h}=\left\{L_{h 1}, L_{h 2} \cdots L_{h P}\right\}$;

- Compute the model vectors associated with the set $\sigma_{h}$ and $L_{h}$, and arrange them into a matrix form as follows:

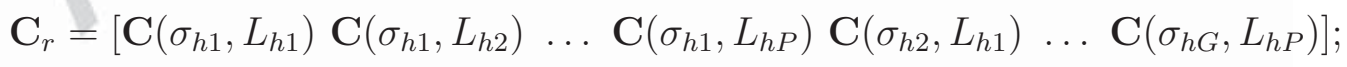

- Decide where to place the "virtual elements" of the interpolation matrix. $\mathbf{C}_{v}=$ $\left[\hat{\mathbf{C}}\left(\sigma_{h 1}, L_{h 1}\right) \hat{\mathbf{C}}\left(\sigma_{h 1}, L_{h 2}\right) \ldots \hat{\mathbf{C}}\left(\sigma_{h 1}, L_{h P}\right) \hat{\mathbf{C}}\left(\sigma_{h 2}, L_{h 1}\right) \ldots \hat{\mathbf{C}}\left(\sigma_{h G}, L_{h P}\right)\right], \hat{\mathbf{C}}\left(\sigma_{h}, L_{h}\right)$ has a uniform linear frequency behaviour. 
- Find the transformation matrix $\mathbf{B}$ by a least squares solution of $\mathbf{B C}_{r}=\mathbf{C}_{v}$. The "best" interpolation matrix $\mathbf{C}_{v}$ is the one which will minimize $\| \mathbf{B} \mathbf{C}_{r}-$ $\mathbf{C}_{v} \|^{2}$.

where $\mathbf{k}_{10}=\widehat{\mathbf{A}}\left(t_{2}-t_{1}\right) \mathbf{k}$ is another eigenvector. Similarly, $\mathbf{k}_{20}=\widehat{\mathbf{A}}\left(t_{1}-t_{2}\right) \mathbf{k}$ is the second eigenvector for $t=t_{2} . \mathbf{k}_{10}$ and $\mathbf{k}_{20}$ are complex valued and not collinear with 
$\mathbf{k}$, any non-zero coefficients linear combination of $\mathbf{k}$ and $\mathbf{k}_{10}$ or $\mathbf{k}$ and $\mathbf{k}_{20}$ is complex valued. Therefore, $\boldsymbol{\Phi}(t)$ has only one real eigenvector $(\mathbf{k})$ corresponding to one single zero eigenvalue, and $\operatorname{real}\left\{\mathbf{k}^{T} \boldsymbol{\Phi}(t) \mathbf{k}\right\}=\mathbf{k}^{T} \operatorname{real}\{\boldsymbol{\Phi}(t)\} \mathbf{k}=0$, only one solution for $t_{1}$ or $t_{2}$. Thus, the number of zero eigenvalue of $\operatorname{real}\left\{\boldsymbol{\Phi}\left(t_{1}\right)\right\}$ and $\operatorname{real}\left\{\boldsymbol{\Phi}\left(t_{2}\right)\right\}$ is 1 . case $2: t \neq t_{1}, t_{2}$ and $t_{3}$. $\boldsymbol{\Phi}(t)$ has 2 zero eigenvalues, we can find easily 2 non-linearly correlated eigenvectors $\mathbf{k}_{1}$ and $\mathbf{k}_{2}$ corresponding to the zero eigenvalues:

$$
\begin{aligned}
& \mathbf{k}_{1}^{H} \boldsymbol{\Phi}(t) \mathbf{k}_{1}=\mathbf{k}_{1}^{H} \widehat{\mathbf{A}}^{H}(t) \mathbf{U}_{N} \mathbf{U}_{N}^{H} \widehat{\mathbf{A}}(t) \mathbf{k}_{1}=0 \\
& \mathbf{k}_{2}^{H} \boldsymbol{\Phi}(t) \mathbf{k}_{2}=\mathbf{k}_{2}^{H} \widehat{\mathbf{A}}^{H}(t) \mathbf{U}_{N} \mathbf{U}_{N}^{H} \widehat{\mathbf{A}}(t) \mathbf{k}_{2}=0
\end{aligned}
$$

where $\mathbf{k}_{1}=\widehat{\mathbf{A}}\left(t_{1}-t\right) \mathbf{k}$ and $\mathbf{k}_{2}=\widehat{\mathbf{A}}\left(t_{2}-t\right) \mathbf{k}$. Due to $t \neq t_{1}, t_{2}$ and $t_{3}, \mathbf{k}_{1}$ and $\mathbf{k}_{2}$ are complex and non-linearly correlated. For these values of $t$, we can show that any linear combination of $\mathbf{k}_{1}$ and $\mathbf{k}_{2}$ will always be complex valued. $\boldsymbol{\Phi}(t)$ has no real eigenvector corresponding to zero eigenvalue. Then, $\mathbf{k}^{T} \boldsymbol{\Phi}(t) \mathbf{k} \neq 0$, $\operatorname{real}\left\{\mathbf{k}^{T} \boldsymbol{\Phi}(t) \mathbf{k}\right\}=\mathbf{k}^{T} \operatorname{real}\{\boldsymbol{\Phi}(t)\} \mathbf{k} \neq 0$, which means $\operatorname{real}\{\boldsymbol{\Phi}(t)\}$ is full rank, there is no zero eigenvalue.

case 3: $t=t_{3}$. When $t=t_{1}$ and $t_{2}$, We have:

$$
\begin{aligned}
\mathbf{k}^{T} \boldsymbol{\Phi}\left(t_{1}\right) \mathbf{k} & =\mathbf{k}^{T} \widehat{\mathbf{A}}^{H}\left(t_{1}\right) \mathbf{U}_{N} \mathbf{U}_{N}^{H} \widehat{\mathbf{A}}\left(t_{1}\right) \mathbf{k}=0 \\
\mathbf{k}^{T} \boldsymbol{\Phi}\left(t_{2}\right) \mathbf{k} & =\mathbf{k}^{T} \widehat{\mathbf{A}}^{H}\left(t_{2}\right) \mathbf{U}_{N} \mathbf{U}_{N}^{H} \widehat{\mathbf{A}}\left(t_{2}\right) \mathbf{k}=0
\end{aligned}
$$


which are equivalent to

$$
\begin{aligned}
\mathbf{U}_{N}^{H} \widehat{\mathbf{A}}\left(t_{1}\right) \mathbf{k} & =0 \\
\mathbf{U}_{N}^{H} \widehat{\mathbf{A}}\left(t_{2}\right) \mathbf{k} & =0
\end{aligned}
$$

For $t=t_{3}$, any linear combination of above equations leads $\mathbf{U}_{N}^{H} \widehat{\mathbf{A}}\left(t_{1}\right) \mathbf{k}+\alpha \mathbf{U}_{N}^{H} \widehat{\mathbf{A}}\left(t_{2}\right) \mathbf{k}=$ $\mathbf{U}_{N}^{H} \widehat{\mathbf{A}}\left(t_{3}\right)\left\{\widehat{A}^{H}(\Delta t)+\alpha \widehat{\mathbf{A}}(\Delta t)\right\} \mathbf{k}=0$. Only when $\alpha$ is equal to 1 or $-1, \widehat{A}^{H}(\Delta t)+$ $\alpha \widehat{\mathbf{A}}(\Delta t)$ is a pure real or imaginary matrix.

For $\alpha=1, \mathbf{U}_{N}^{H} \widehat{\mathbf{A}}\left(t_{3}\right)\left\{\widehat{A}^{H}(\Delta t)+\widehat{\mathbf{A}}(\Delta t)\right\} \mathbf{k}=2 \mathbf{U}_{N}^{H} \widehat{\mathbf{A}}\left(t_{3}\right) \operatorname{real}\{\widehat{A}(\Delta t)\} \mathbf{k}=2 \mathbf{U}_{N}^{H} \widehat{\mathbf{A}}\left(t_{3}\right) \mathbf{k}_{3}$.

For $\alpha=-1, \mathbf{U}_{N}^{H} \widehat{\mathbf{A}}\left(t_{3}\right)\left\{\widehat{A}^{H}(\Delta t)-\widehat{\mathbf{A}}(\Delta t)\right\} \mathbf{k}=2 j \mathbf{U}_{N}^{H} \widehat{\mathbf{A}}\left(t_{3}\right) \operatorname{imag}\{\widehat{A}(\Delta t)\} \mathbf{k}=2 j \mathbf{U}_{N}^{H} \widehat{\mathbf{A}}\left(t_{3}\right) \mathbf{k}_{4}$.

Therefore, $\mathbf{k}_{3}^{T} \boldsymbol{\Phi}\left(t_{3}\right) \mathbf{k}_{3}=\mathbf{k}_{4}^{T} \boldsymbol{\Phi}\left(t_{3}\right) \mathbf{k}_{4}=0$ with $\mathbf{k}_{3}=\operatorname{real}\{\widehat{A}(\Delta t)\} \mathbf{k}$ and $\mathbf{k}_{4}=\operatorname{imag}\{\widehat{A}(\Delta t)\} \mathbf{k}$.

In addition, $\mathbf{a}^{H}(t) \mathbf{U}_{N} \mathbf{U}_{N}^{H} \mathbf{a}(t)$ is always real valued, thus, $t_{3}$ is a solution of $\lambda_{\min }(t)=0$ and $\operatorname{real}\left\{\boldsymbol{\Phi}\left(t_{3}\right)\right\}$ only two zero eigenvalues with corresponding eigenvectors $\mathbf{k}_{3}$ and $\mathbf{k}_{4}$. When the number of echoes is superior to 2 , the number of zero eigenvalues of $\operatorname{real}\{\boldsymbol{\Phi}(t)\}$ corresponding to the true time delay is odd. For false time delay, this number is even.

\section{Appendix $\mathrm{C}$}

In this appendix, we present MLE for the roughness parameters estimation. The time delays are estimated ( $\hat{t}_{k}$ is the $k$ th estimated time delay) and the noise being a Gaussian white noise with zero mean and variance $\sigma^{2}$. Referring to Eq. (1), the joint probability density function for all observations can be calculated:

$$
f\left(\mathbf{r}, b_{k}\right)=\frac{1}{\left(\pi \sigma^{2}\right)^{N}} \exp \left\{-\frac{\sum_{i=1}^{N}\left|r\left(f_{i}\right)-\sum_{k=1}^{K} s_{k} e\left(f_{i}\right) \exp \left(-j 2 \pi f_{i} \hat{t}_{k}-b_{k} f_{i}^{2}\right)\right|^{2}}{\sigma^{2}}\right\}
$$


In practice, MLE is obtained by maximizing a log-likelihood function $L\left(\mathbf{r}, b_{k}\right)$ instead of the joint density function $f\left(\mathbf{r}, b_{k}\right)$ as follows:

$$
\begin{aligned}
L\left(\mathbf{r}, b_{k}\right) & =\ln f\left(\mathbf{r}, b_{k}\right) \\
& =-\frac{\sum_{i=1}^{N}\left|r\left(f_{i}\right)-\sum_{k=1}^{K} s_{k} e\left(f_{i}\right) \exp \left(-j 2 \pi f_{i} \hat{t}_{k}-b_{k} f_{i}{ }^{2}\right)\right|^{2}}{\sigma^{2}}-N \ln \left(\pi \sigma^{2}\right)
\end{aligned}
$$

The optimal estimation (for the roughness parameters $b_{k}$ ) is obtained by finding the solution of $\frac{\partial L\left(\mathbf{r}, b_{k}\right)}{\partial b_{k}}=0$.

\section{Acknowledgment}

The authors would like to thank the China Scholarship Council (No. 201306150010) and the grant of science and technology planning project of Guangdong (No. 2015A050502011) for funding part of this work. This work may contribute to COST Action TU1208 "Civil Engineering Applications of Ground Penetrating Radar".

\section{References}

[1] A.P. Annan, N. Diamanti, J.D. Redman, S.R. Jackson, Ground-penetrating radar for assessing winter roads, Geophysics 81 (2016) WA101-WA109.

[2] A. Benedetto, L. Pajewski, Civil Engineering Applications of Ground Penetrating Radar, Springer International Publishing, Switzerland, 2015.

[3] A.S. Venkatachalam, X. Xu, D. Huston, T. Xia, Development of a new high speed dual-channel impulse ground penetrating radar, IEEE Journal of Selected Topics in Applied Earth Observations and Remote Sensing 7 (2014) 753-760. 
[4] D.H. Chen, F. Hong, W. Zhou, P. Ying, Estimating the hotmix asphalt air voids from ground penetrating radar, NDT \& E International 68 (2014) 120-127.

[5] H. Liu, M. Sato, In situ measurement of pavement thickness and dielectric

[12] C. Le Bastard, V. Baltazart, Y. Wang, J. Saillard, Thin-pavement thickness 
estimation using GPR with high-resolution and super resolution methods, IEEE Transactions on Geoscience and Remote Sensing 45 (August 2007) 2511-2519.

[13] N. Pinel, C. Le Bastard, V. Baltazart, C. Bourlier, Y. Wang, Influence of layer roughness for road survey by ground penetrating radar at nadir: theoretical study, IET Radar, Sonar \& Navigation 5 (July 2011) 650-656.

[14] K. Luo, A. Manikas, Superresolution multitarget parameter estimation in MIMO radar, IEEE Transactions on Geoscience and Remote Sensing 51 (6) (2013) 3683-3693.

[15] D. Kurrant, E. Fear, Technique to decompose near-field reflection data generated from an object consisting of thin dielectric layers, IEEE Transactions on Antennas and Propagation 60 (8) (2012) 3684-3692.

[16] C. Le Bastard, V. Baltazart, Y. Wang, Modified ESPRIT (M-ESPRIT) algorithm for time delay estimation in both any noise and any radar pulse context by a GPR radar, Signal Processing 90 (2010) 173-179.

[17] K. Chahine, V. Baltazart, Y. Wang, Interpolation-based matrix pencil method for parameter estimation of dispersive media in civil engineering, Signal Processing 90 (2010) 2567-2580.

[18] N. Pinel, C. Le Bastard, C. Bourlier, M. Sun, Asymptotic Modeling of Coherent Scattering from Random Rough Layers: Application to Road Survey by GPR at Nadir, Int. Journal of Antennas and Propagation (2012) Article ID 874840.

[19] M. Sun, N. Pinel, C. Le Bastard, V. Baltazart, A. Ihamouten, Y. Wang, Time 
delay and interface roughness estimation by subspace algorithms for pavement survey by radar, Near Surface Geophysics 13 (June 2015) 279-287.

[20] M. Sun, C. Le Bastard, N. Pinel, Y. Wang, J. Li, Road surface layers geometric parameters estimation by ground penetrating radar using Estimation of Signal Parameters via Rotational Invariance Techniques method, IET Radar, Sonar \& Navigation 10 (2016) 603-609.

[21] L. Qu, Q. Sun, T. Yang, L. Zhang, Y. Sun, Time-delay estimation for ground penetrating radar using ESPRIT with improved spatial smoothing preprocessing, IEEE Geoscience and remote sensing letters 11 (2014) 1315-1319.

[22] A. Schatzberg, A. J. Devaney, A. J. Witten, Estimating target location from scattered field data, Signal Processing 40 (1994) 227-237.

[23] N. Déchamps, N. de Beaucoudrey, C. Bourlier, S. Toutain, Fast numerical method for electromagnetic scattering by rough layered interfaces: Propagationinside-layer expansion method, J. Opt. Soc. Am. A. 23 (2006) 359-369.

[24] C. Bourlier, G. Kubické, N. Déchamps, Fast method to compute scattering by a buried object under a randomly rough surface: PILE combined with FB-SA, J. Opt. Soc. Am. A. 5 (2009) 260-263.

[25] C. Bourlier, C. Le Bastard, V. Baltazart, Generalization of PILE method to the EM scattering from stratified subsurface with rough interlayers: application to the detection of debondings within pavement structure, IEEE Transactions on Geoscience and Remote Sensing 53 (2015) 4104-4115. 
[26] F. Koudogbo, P. F. Combes, H. J. Mametsa, Numerical and experimental validations of IEM for bistatic scattering from natural and manmade rough surfaces, Progress In Electromagnetics Research 46 (2004) 203-244.

[27] E. Li, K. Sarabandi, Low grazing incidence millimeter-wave scattering models and measurements for various road surfaces, IEEE Transactions on Antennas and Propagation 47 (1999) 851-861.

[28] C. Fauchard, Utilisation de radars très hautes fréquences: application à lauscultation non destructive des chaussées, PhD thesis, University of Nantes, France, 2001.

[29] D. Daniel, Ground Penetrating Radar, IEE Press, London, 2nd edn, 2004.

[30] X. Li, R. Wu, an efficient algorithm for time delay estimation, IEEE Transactions on Signal Processing 46 (1998) 2231-2235.

[31] M. Sun, C. Le Bastard, Y. Wang, N. Pinel, Time delay estimation using ESPRIT with extended improved spatial smoothing techniques for radar signals, IEEE Geoscience and Remote Sensing Letters 13 (2016) 73-77.

[32] B. Friedlander, A. J. Weiss, Direction finding using spatial smoothing with interpolated arrays, IEEE Transactions on Aerospace and Electronic Systems 28 (1982) 574-587.

[33] A. J. Weiss, B. Friedlander, performance analysis of spatial smoothing with interpolated arrays, IEEE Transactions on Signal Processing 41 (1993) 18811892. 
[34] S. Marcos, J. Sanchez-Araujo, Méthodes linéaires haute résolution pour l'estimation de directions d'arrivée de sources. Performances asymptotiques et complexité, Traitement du Signal 14 (1997) 99-116.

[35] T. J. Shan, M. Wax, T. Kailath, On spatial smoothing for direction-of-arrival estimation of coherent signals, IEEE Transactions on Acoustics, Speech and Signal Processing 33 (1985) 806-811.

[36] F. Ge, D. Shen, Y. Peng, V.O.K. Li, Super-Resolution Time Delay Estimation in Multipath Environments, IEEE Transactions on Circuits and Systems 54 (2007) 1977-1986.

[37] F. Ge, Q. Wan , X. Wang, Y. Peng, Frequency estimation of the sinusoidal signals with lowpass envelopes based on the eigenanalysis, in IEEE Radar Conf. (2002) 453-458. 


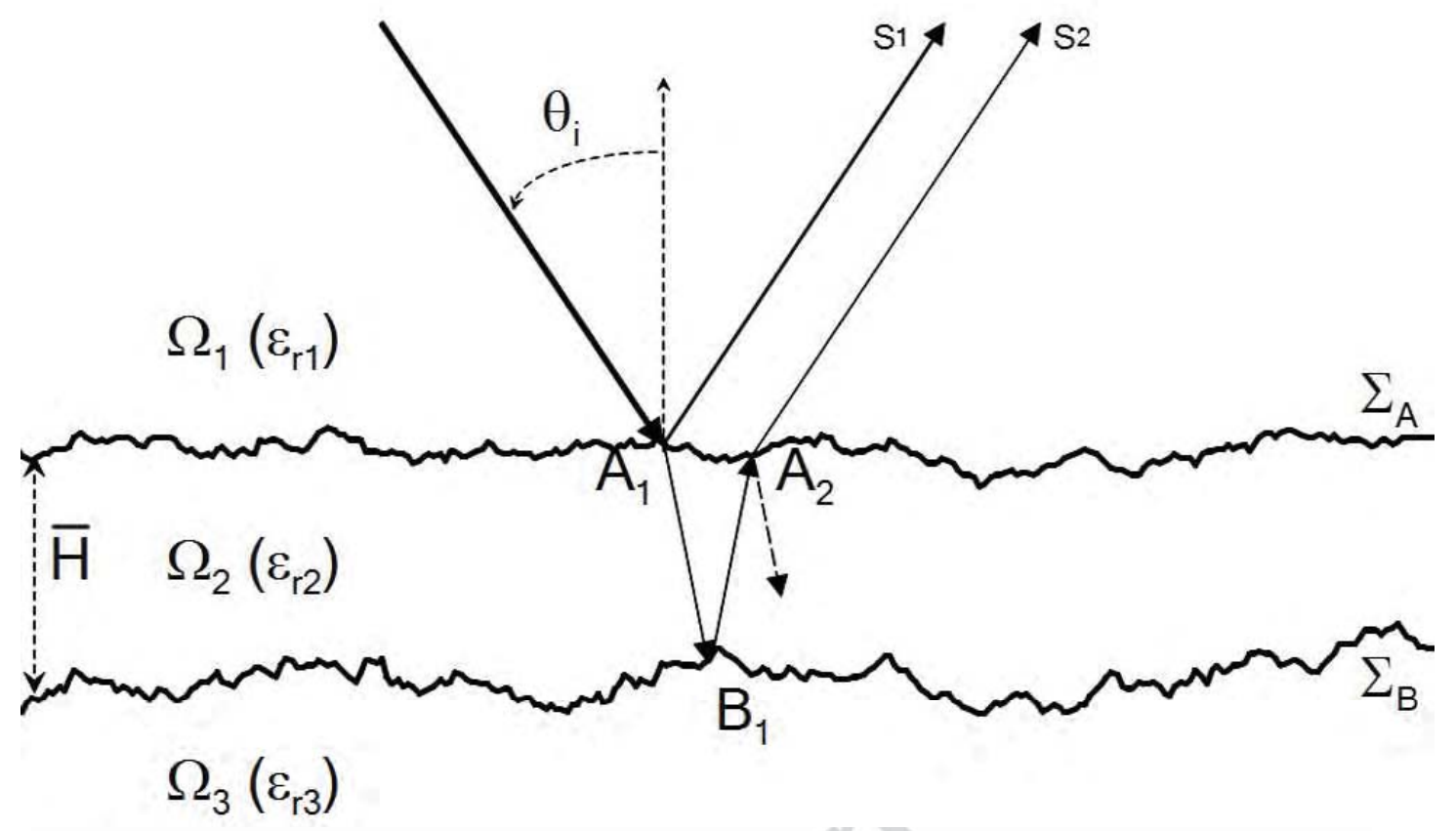

Figure 1: Rough Pavement Configuration. 

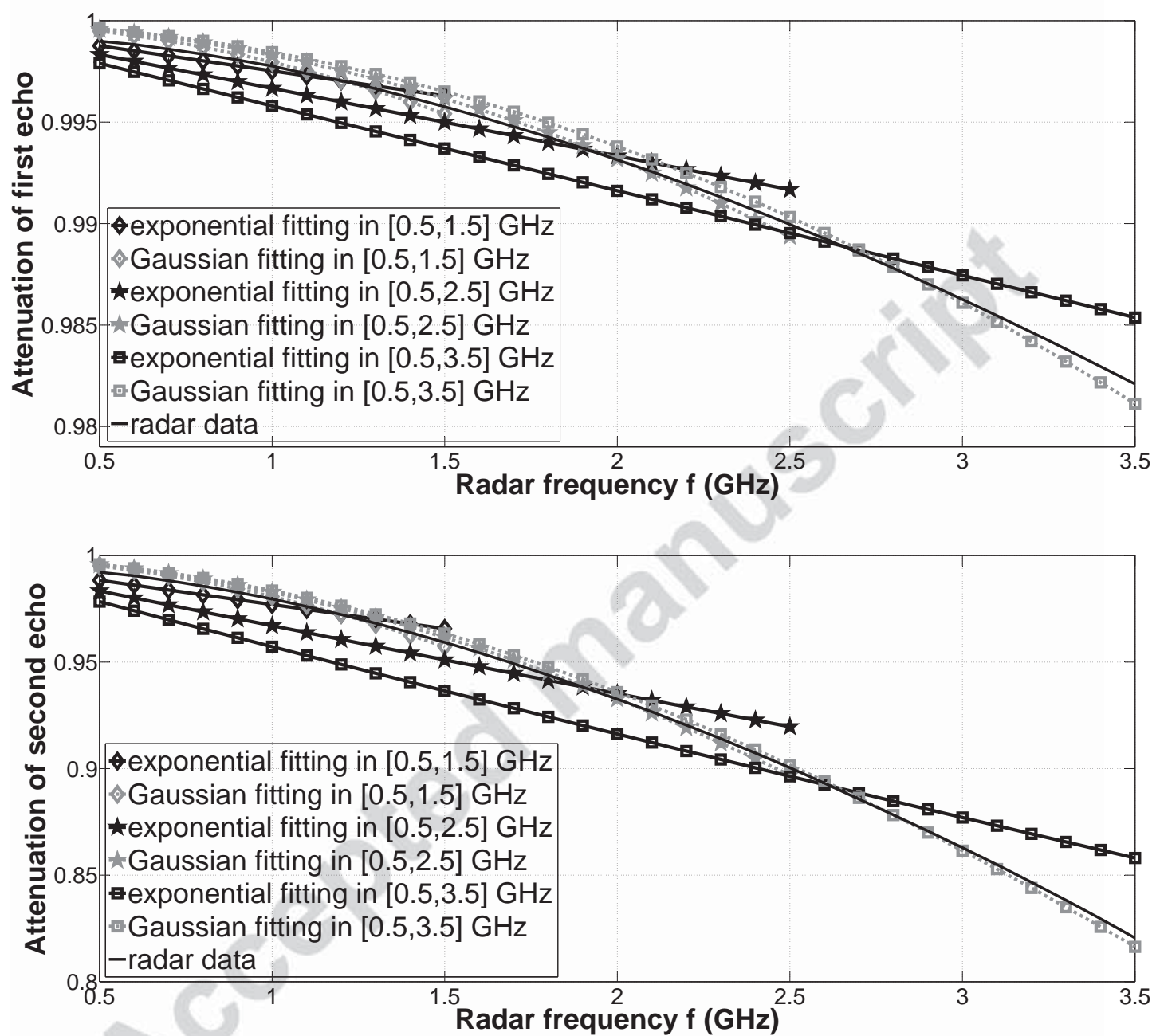

Figure 2: Frequency behaviour of echoes with curve fitting results in $[0.5,1.5] \mathrm{GHz},[0.5,2.5] \mathrm{GHz}$ and $[0.5,3.5] \mathrm{GHz}$. 

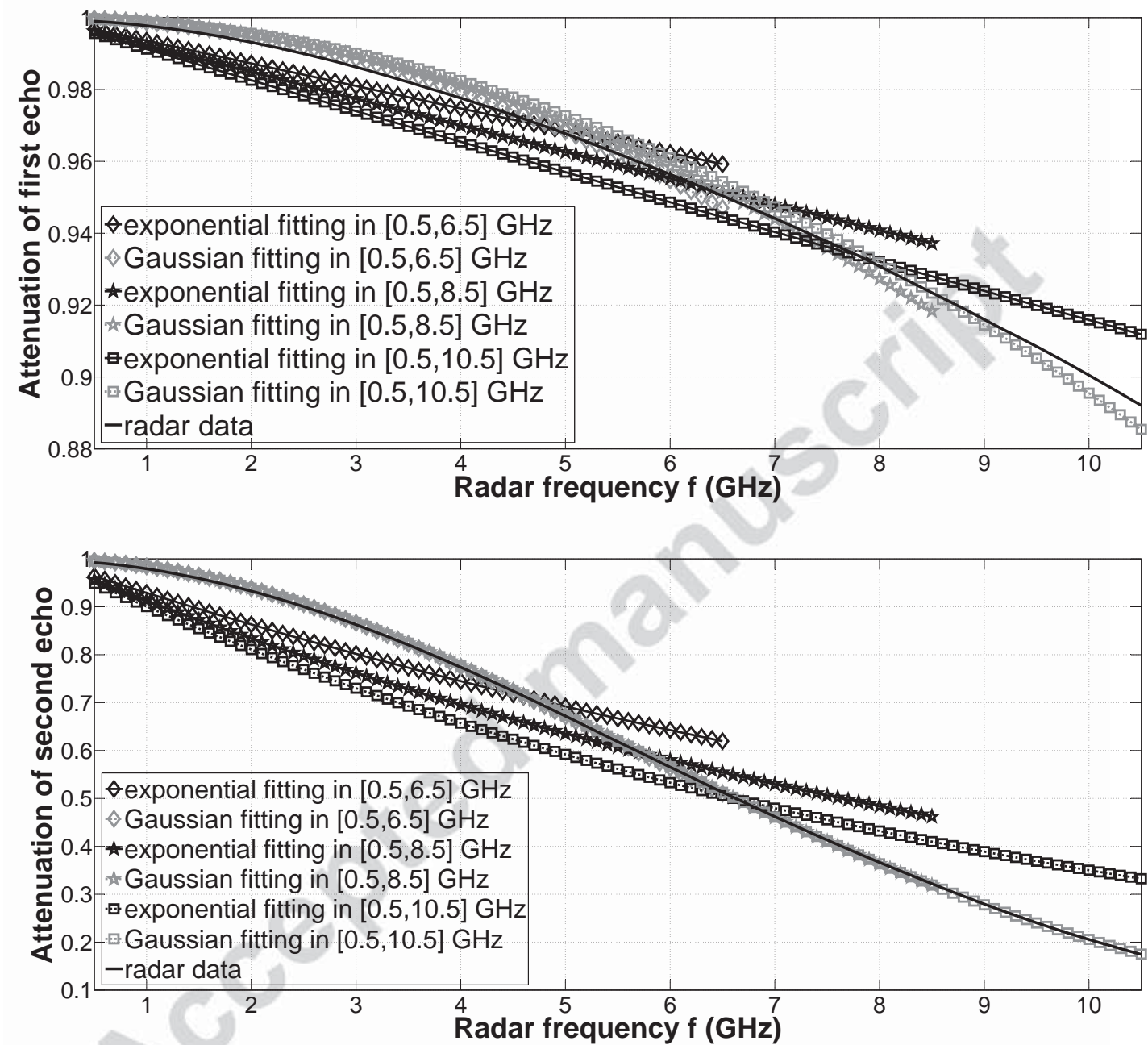

Figure 3: Frequency behaviour of echoes with curve fitting results in $[0.5,6.5] \mathrm{GHz},[0.5,8.5] \mathrm{GHz}$ and $[0.5,10.5] \mathrm{GHz}$. 


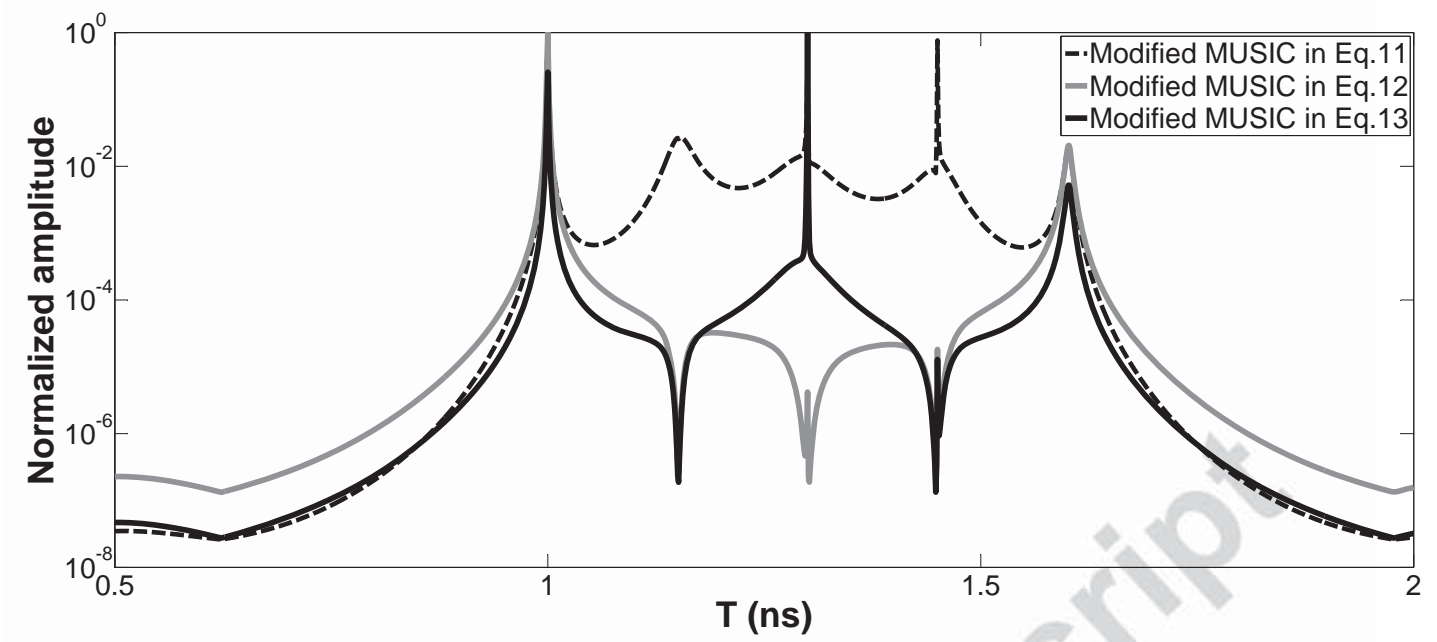

Figure 4: Pseudo-spectrum of MUSIC for time delay estimation with $\mathrm{SNR}=30 \mathrm{~dB}$, the three time delays are $1 \mathrm{~ns}, 1.3 \mathrm{~ns}$ and $1.6 \mathrm{~ns}$.

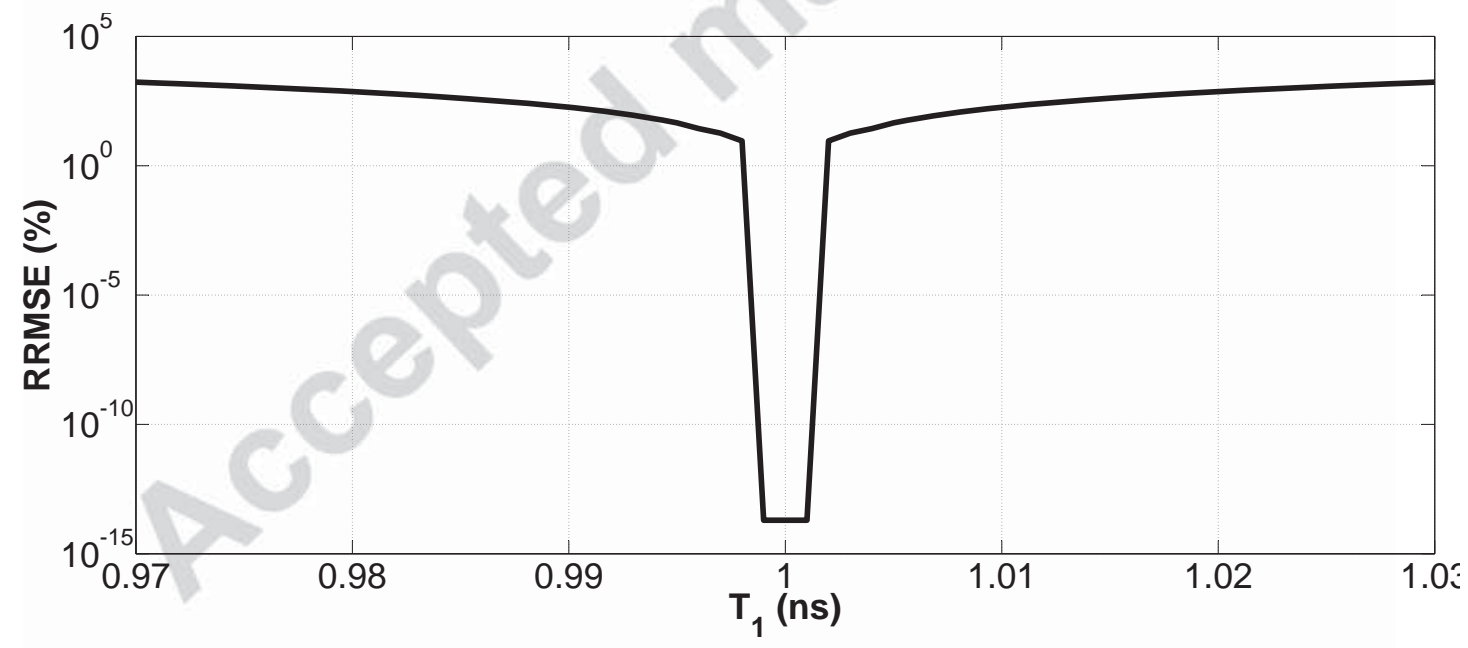

Figure 5: RRMSE on the estimated roughness parameter $b_{1}=1.10 \times 10^{-3} \mathrm{GHz}^{-2}\left(\sigma_{h A}=0.5 \mathrm{~mm}\right.$, $L_{c A}=6.4 \mathrm{~mm}$ ) against first time delay $T_{1}=1 \mathrm{~ns}$ in a noiseless environment by MLE. 


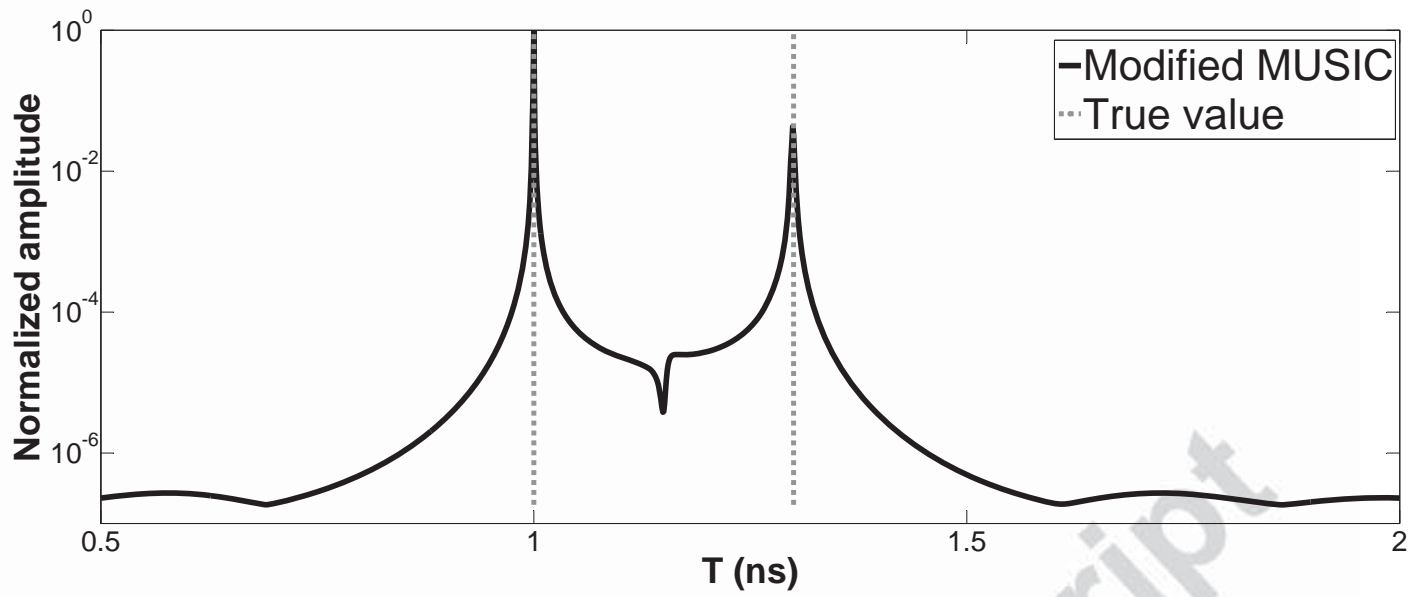

Figure 6: Case 1, Pseudo-spectrum of MUSIC for time delay estimation with $\mathrm{SNR}=20 \mathrm{~dB}$, the two time delays are $1 \mathrm{~ns}$ and $1.3 \mathrm{~ns}$ in grey dashed line, slightly overlapped.

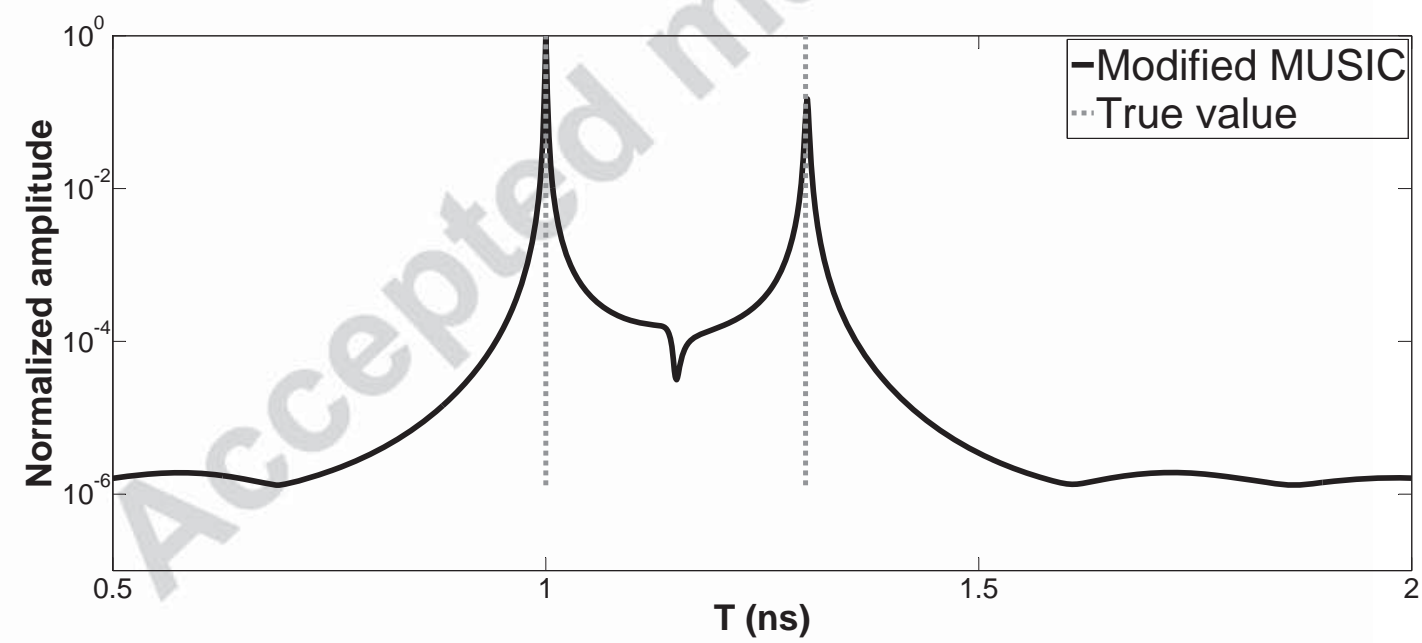

Figure 7: Case 2, Pseudo-spectrum of MUSIC for time delay estimation with $\mathrm{SNR}=20 \mathrm{~dB}$, the two time delays are $1 \mathrm{~ns}$ and $1.3 \mathrm{~ns}$ in grey dashed line, slightly overlapped. 


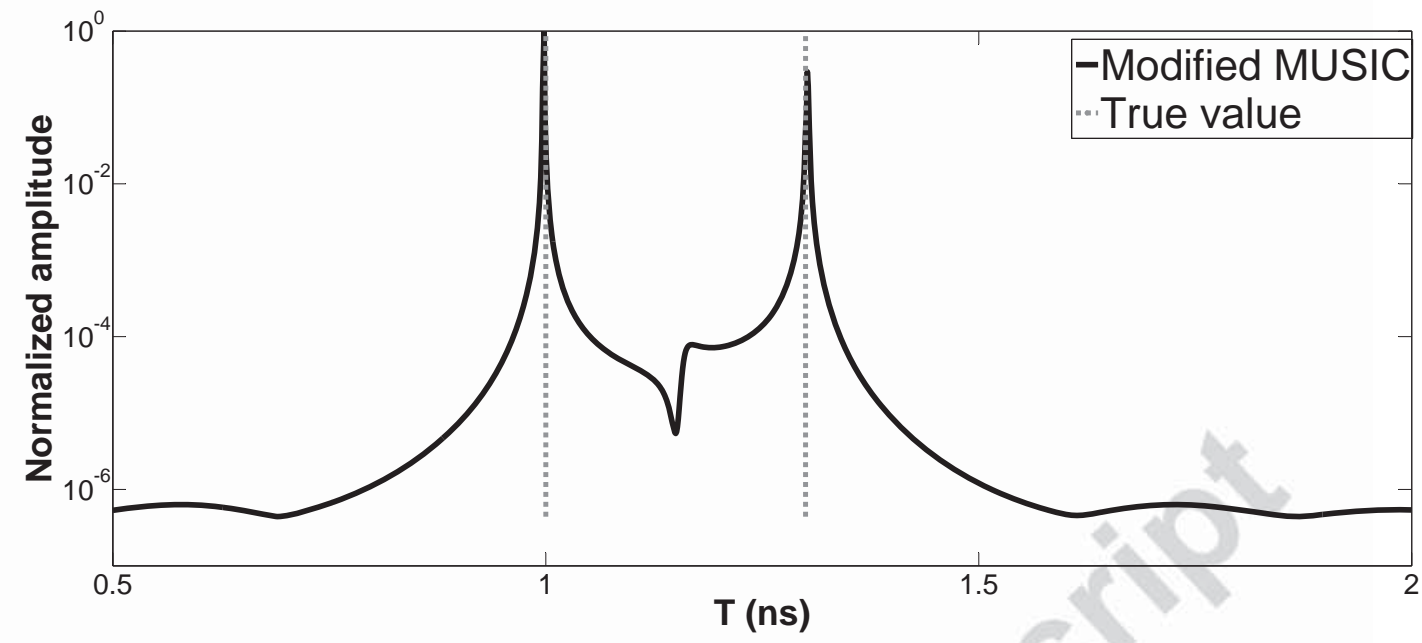

Figure 8: Case 3, Pseudo-spectrum of MUSIC for time delay estimation with $\mathrm{SNR}=20 \mathrm{~dB}$, the two time delays are $1 \mathrm{~ns}$ and $1.3 \mathrm{~ns}$ in grey dashed line, slightly overlapped.

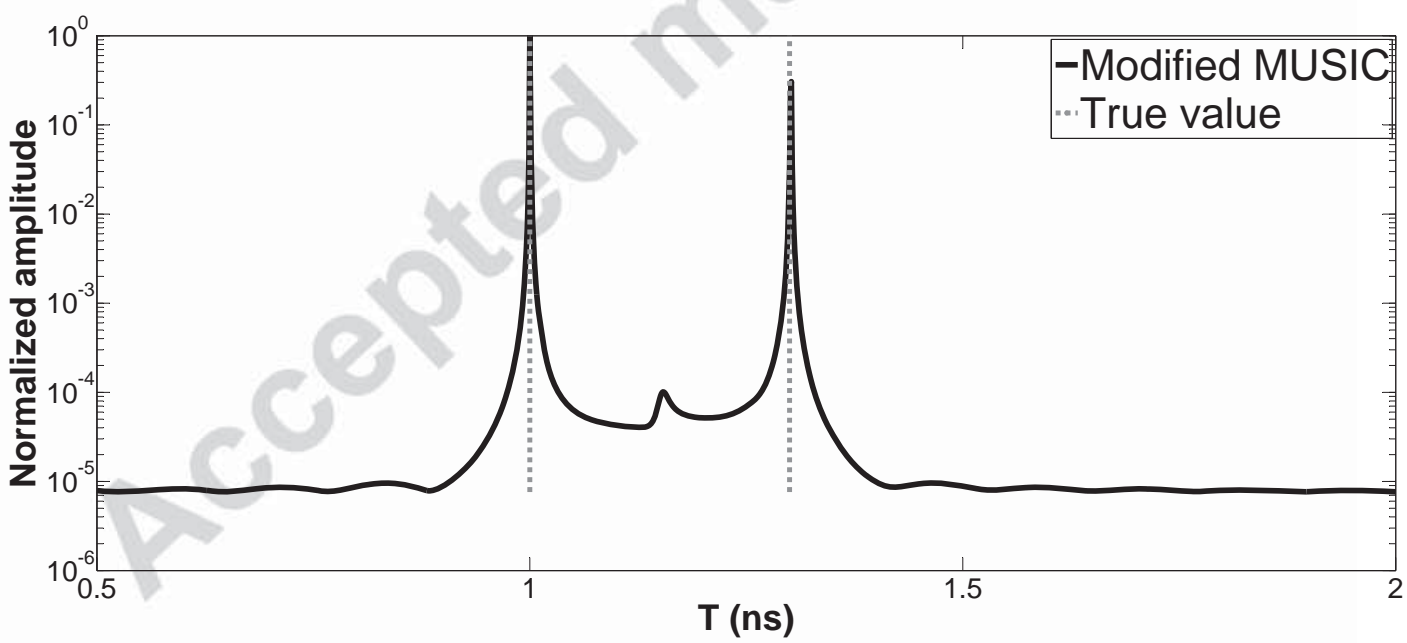

Figure 9: Case 1, Pseudo-spectrum of MUSIC for time delay estimation with $\mathrm{SNR}=20 \mathrm{~dB}$, the two time delays are $1 \mathrm{~ns}$ and $1.3 \mathrm{~ns}$ in grey dashed line, non-overlapped. 


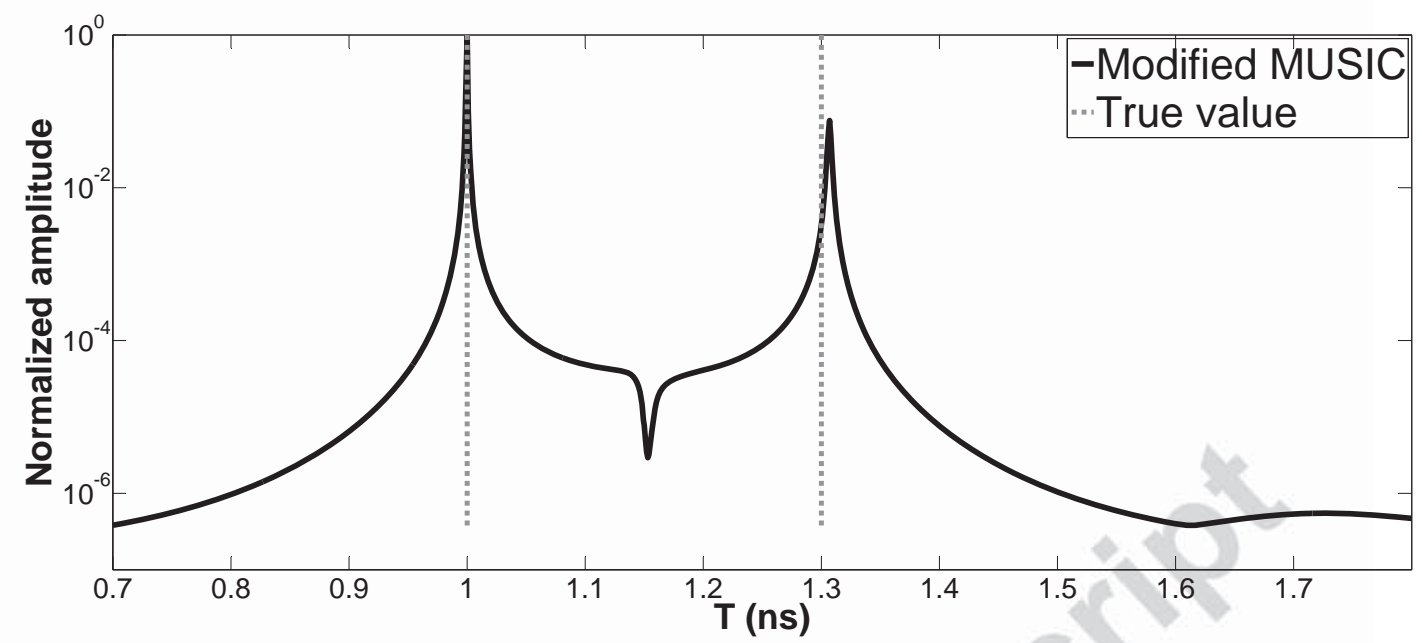

Figure 10: Case 4, Pseudo-spectrum of MUSIC for time delay estimation with SNR=20 dB, the two time delays are $1 \mathrm{~ns}$ and $1.3 \mathrm{~ns}$ in grey dashed line, slightly overlapped, low-loss media.

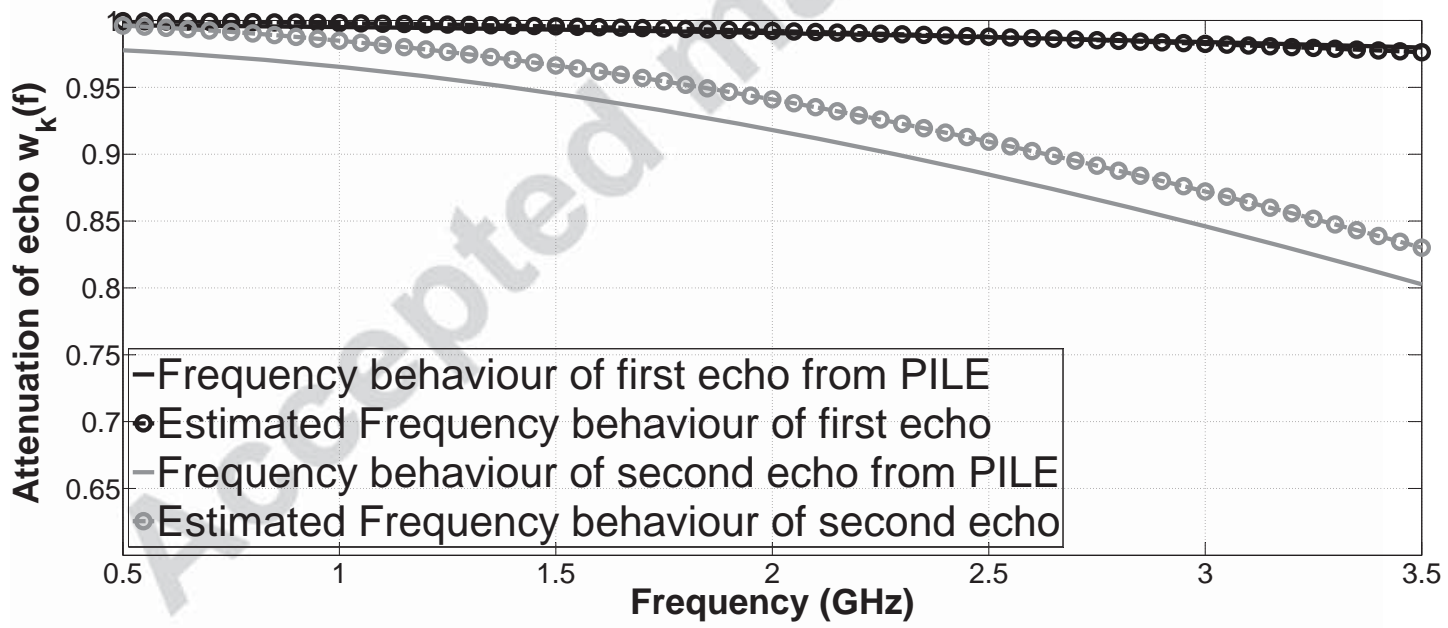

Figure 11: Case 1, Expression for frequency behaviour of backscattered echoes by using estimated roughness parameter versus frequency behaviour of backscattered echoes from radar data, slightly overlapped. 


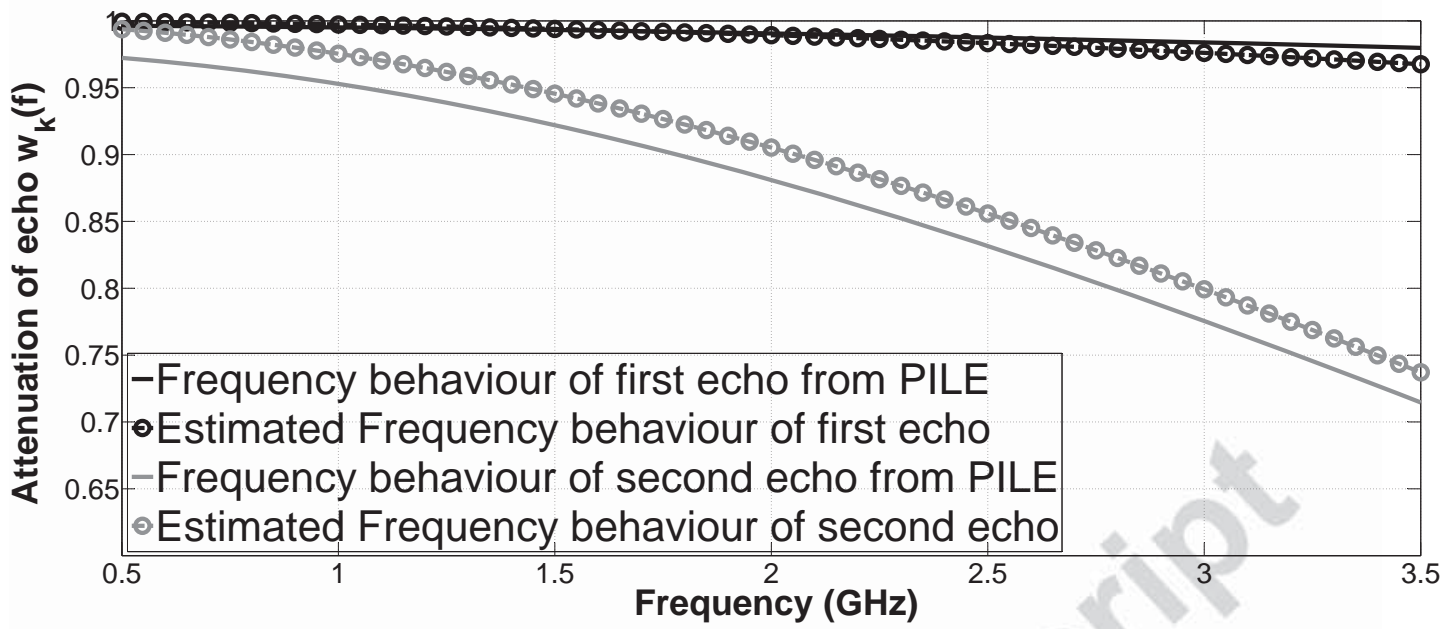

Figure 12: Case 2, Expression for frequency behaviour of backscattered echoes by using estimated roughness parameter versus frequency behaviour of backscattered echoes from radar data, slightly overlapped.

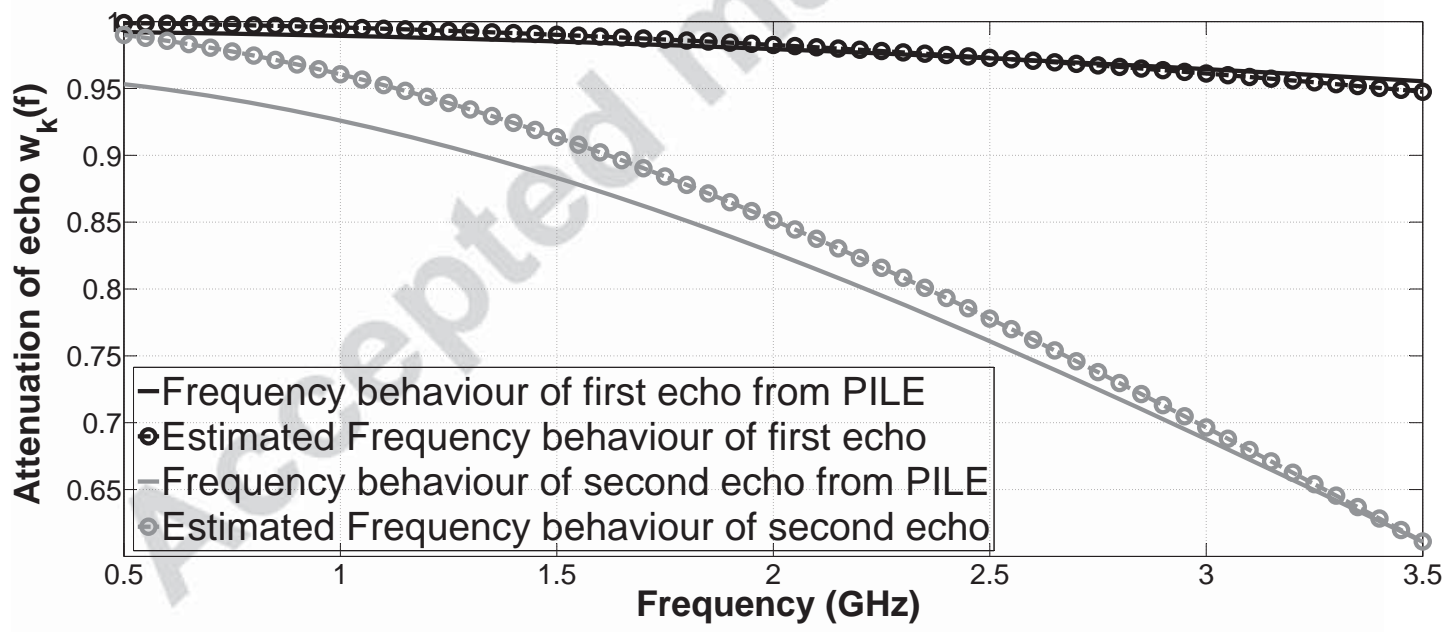

Figure 13: Case 3, Expression for frequency behaviour of backscattered echoes by using estimated roughness parameter versus frequency behaviour of backscattered echoes from radar data, slightly overlapped. 


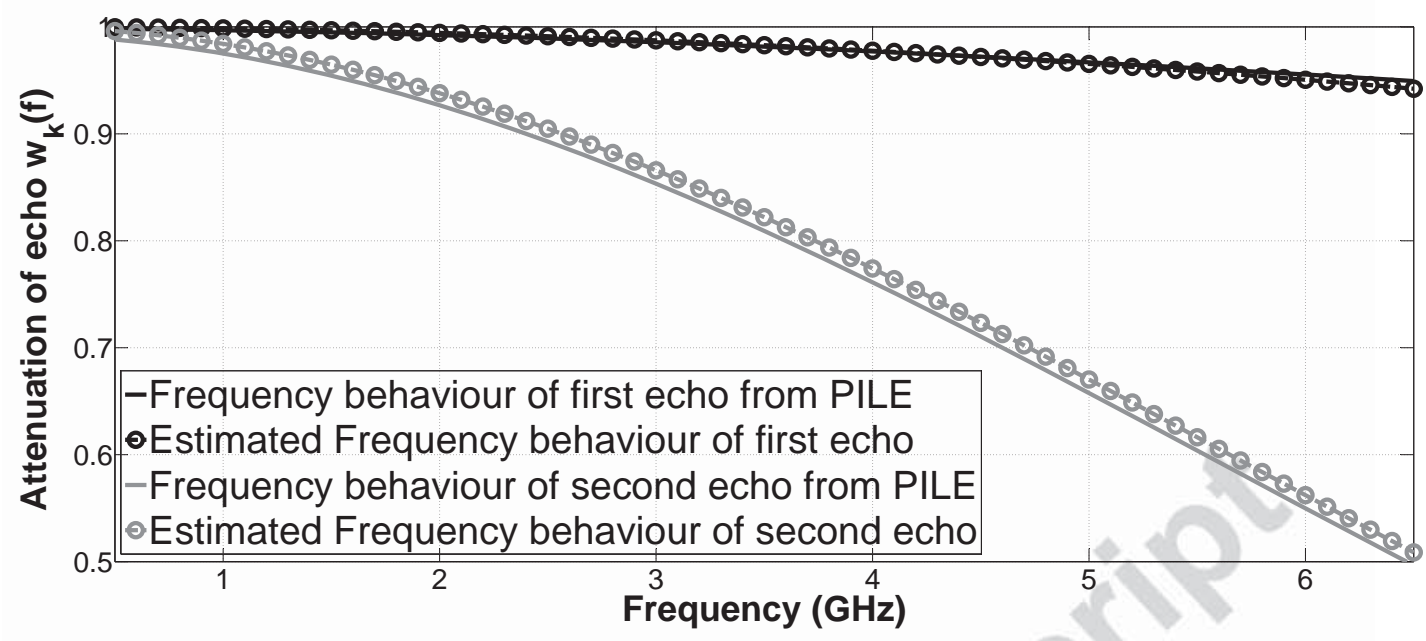

Figure 14: Case 1, Expression for frequency behaviour of backscattered echoes by using estimated roughness parameter versus frequency behaviour of backscattered echoes from radar data, nonoverlapped.

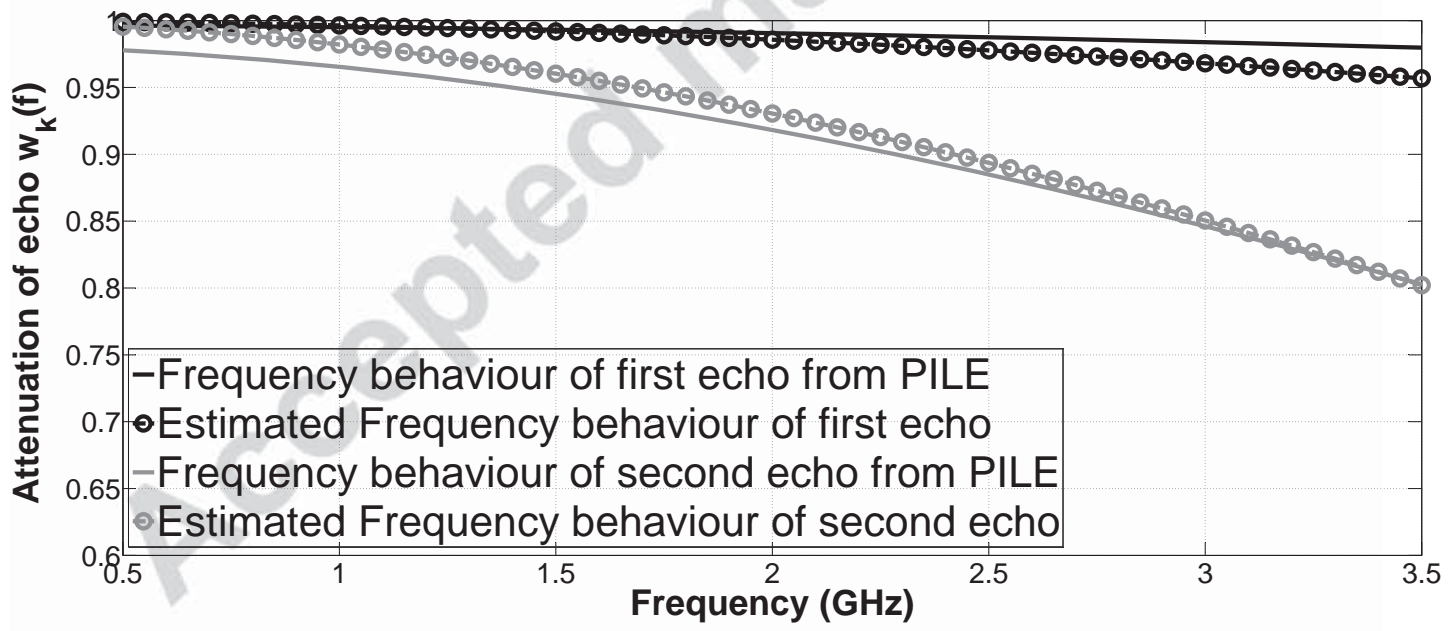

Figure 15: Case 4, Expression for frequency behaviour of backscattered echoes by using estimated roughness parameter versus frequency behaviour of backscattered echoes from radar data, slightly overlapped, low-loss media. 


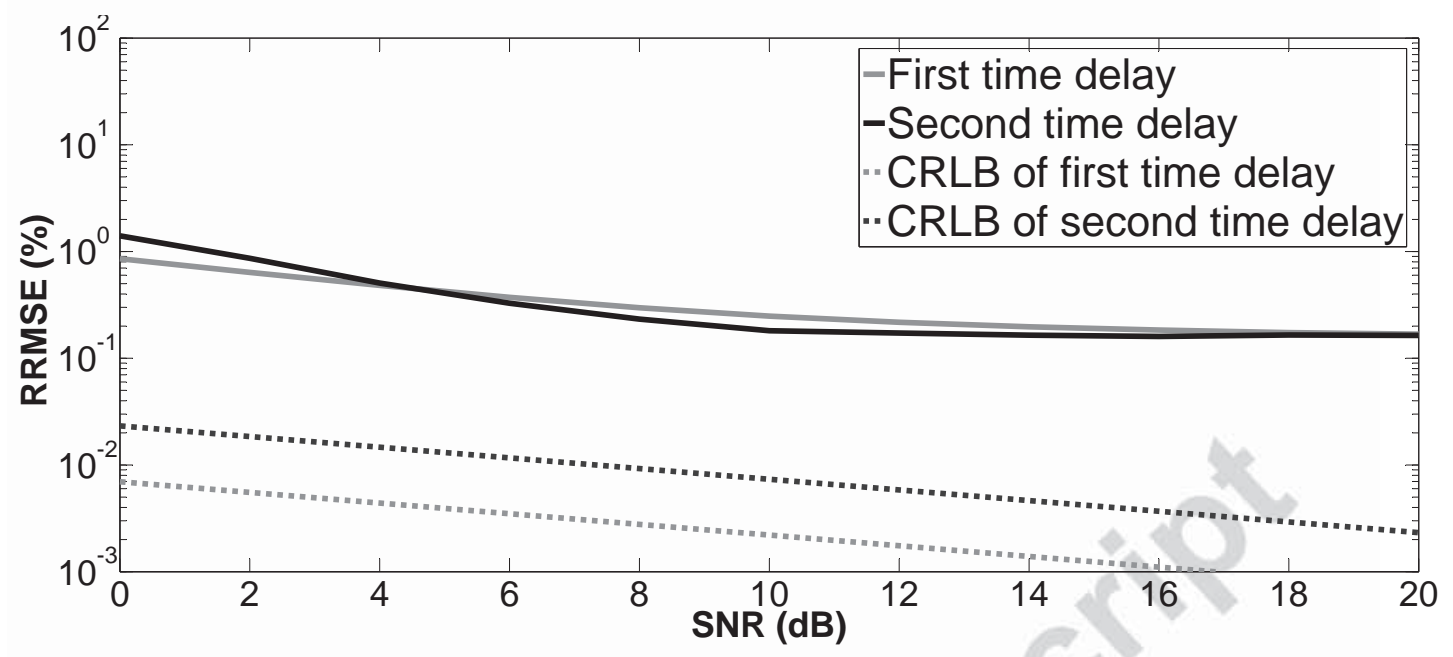

Figure 16: Simulation 2, RRMSE on the estimated time delay $t_{k},(k=1,2)$ vs. SNR after 500 Monte-Carlo simulations for case 1, slightly overlapped.

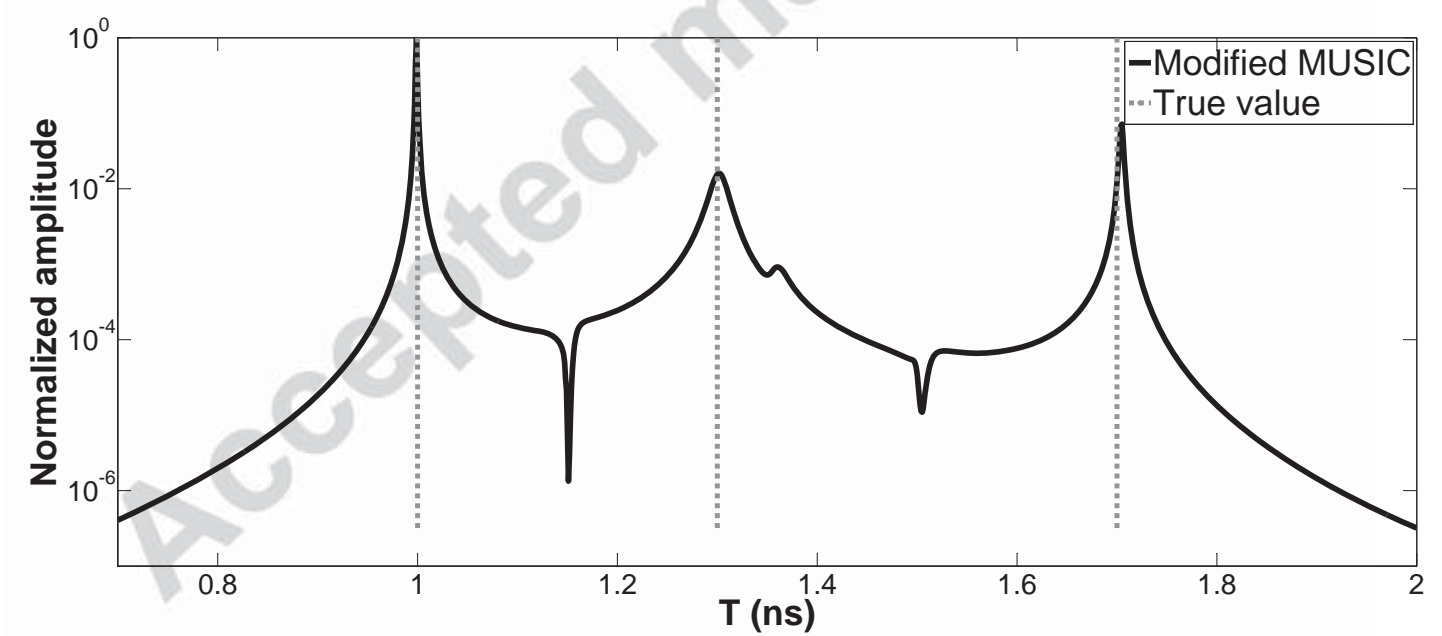

Figure 17: Simulation 3, Pseudo-spectrum of MUSIC for time delay estimation with $\mathrm{SNR}=20 \mathrm{~dB}$, the three time delays are $1 \mathrm{~ns}, 1.3 \mathrm{~ns}$ and $1.7 \mathrm{~ns}$ in grey dashed line. 


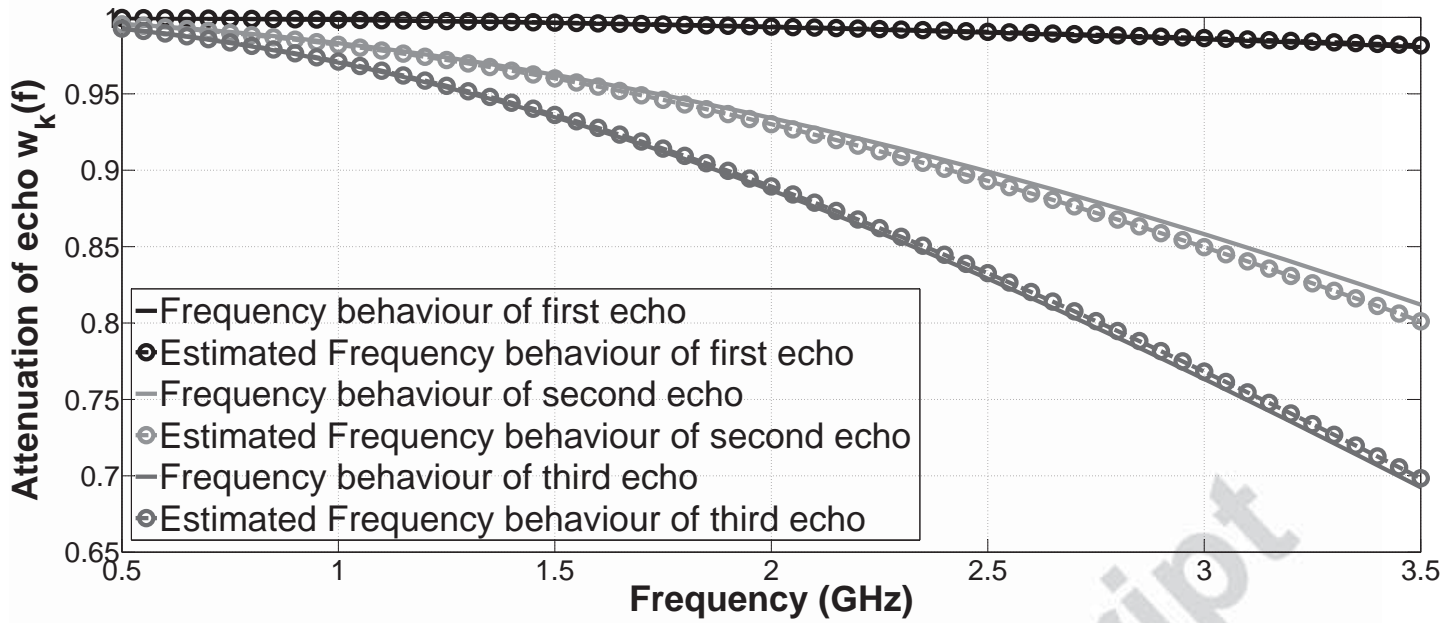

Figure 18: Simulation 3, Expression for frequency behaviour of backscattered echoes by using estimated roughness parameter versus frequency behaviour of backscattered echoes from radar data. 


\begin{tabular}{|c|c|c|}
\hline RMSE \% $\left(s_{1} / s_{2}\right) \backslash$ Model & $|s(f)|=s_{k} \times \exp (-\bar{b} f)$ & $|s(f)|=s_{k} \times \exp \left(-b f^{2}\right)$ \\
\hline Frequency & $0.0290 / 0.371$ & $0.0307 / 0.179$ \\
\hline$[0.5,1.5] G H z$ & $0.0906 / 1.04$ & $0.0433 / 0.257$ \\
\hline$[0.5,2.5] G H z$ & $0.171 / 1.94$ & $0.0605 / 0.324$ \\
\hline$[0.5,3.5] G H z$ & $0.463 / 5.63$ & $0.170 / 0.346$ \\
\hline$[0.5,6.5] G H z$ & $0.698 / 7.99$ & $0.270 / 0.355$ \\
\hline$[0.5,8.5] G H z$ & $0.983 / 9.88$ & $0.367 / 0.323$ \\
\hline$[0.5,10.5] G H z$ & &
\end{tabular}

Table 1: RMSE on curve fitting with Gaussian and exponential functions. 


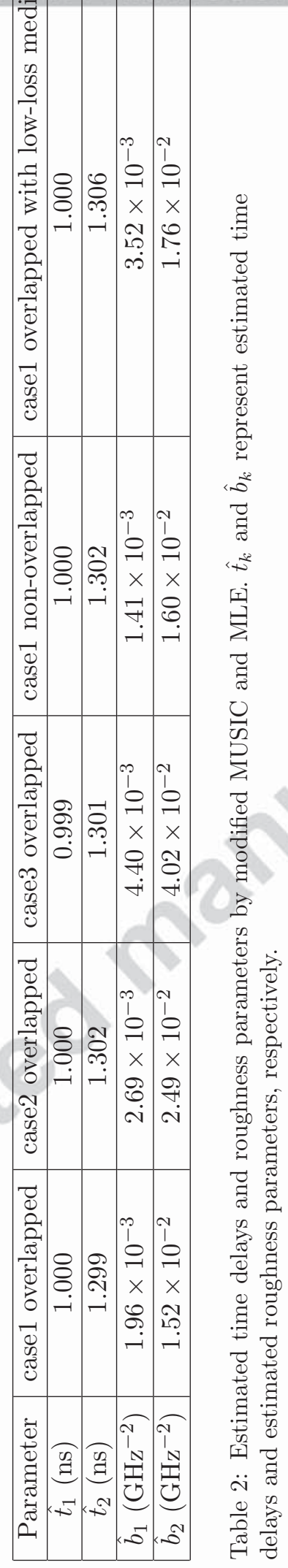

